\title{
Theory of Connexes. I
}

\author{
By
}

Yōhei YAMASAKI*

\section{Introduction}

The classical games Hex and Bridg-it are generalized to Shannon's switching game (on vertices), in which a graph structure is given on a finite set consisting of two edge points and several ordinal points and two players occupy ordinal points alternately. A player called "short" intends to connect the edge points by a sequence of ordinal points which he has occupied and his opponent called "cut" obstruct it. Its game theory has been established more generally in the class of division games, in which we have discussed the game theory without graphs (see Lehman [1] and Yamasaki [2]).

We are going to give a new generalization of the classical games in a purely graph-theoretical argument. Namely, we discuss a pair called "connex" of graph structures over a common set, which naturally yields a division space. In this paper "Theory of connexes. I" we give a general theory of connexes and show that a connex can be essentially embedded into a sphere $S^{2}$ if the given graph structures coincide, except for three cases which are determined completely. In other words, our game theoretical argument characterizes the graphs obtained from simplicial decompositions of $S^{2}$ compatible to the antipodal mapping without triangle besides 2 -simplices.

\section{§1. Preliminary}

Let $\bar{\Pi}$ be a set consisting of three elements $T, \perp$ and $\theta, \Pi$ its subset consisting of $T$ and $\perp$, and ${ }^{\wedge}$ the involution of $\Pi$ without fixed point. We call an element of $\Pi$ a player and $\theta$ nobody. These notion will be fixed throughout this paper.

Communicated by S. Hitotumatu, November 21, 1980.

* Department of Mathematics, Osaka University, Toyonaka 560, Japan. 
Let $X$ and $Y$ be sets and $\pi$ a player. Then we denote by $[X \rightarrow Y]$ the set of mappings from $X$ to $Y$, by $|X|$ or $\# X$ the cardinal number of $X$ and by $i_{Y}^{\pi}$ the mapping from $Y$ to $Y \times \Pi$ given as follows:

$$
i_{Y}^{\pi}(y)=(y, \pi) \text {. }
$$

Let $X$ be a finite set. Then we denote $\mathfrak{P}_{X}=[X \rightarrow \bar{\Pi}]$ and $\mathfrak{D}_{X}=[X \rightarrow \Pi]$. An element $\partial$ of $\mathfrak{P}_{X}$ is called a position and an element $\mathfrak{D}$ of $\mathfrak{D}_{X}$ is called a division. A mapping $\chi^{*}$ from $\mathfrak{D}_{X} \times \Pi$ to $\{1,-1\}$ is called a judge on $X$ if

$$
\sum_{\pi \in \Pi} \chi^{*}(\mathfrak{D}, \pi)=0 \quad \forall_{\mathfrak{D}} \in \mathfrak{D}_{X} .
$$

A pair $\mathscr{D}^{*}=\left(X, \chi^{*}\right)$ of a finite set $X$ and a judge $\chi^{*}$ on it is called a division space.

Let $\mathscr{D}^{*}=\left(X, \chi^{*}\right)$ be a division space and $\partial$ a position on $X$. Then we define another division space $\mathscr{D}_{\partial}^{*}=\left(X_{\partial}, \chi_{\partial}^{*}\right)$ as follows:

$$
\begin{aligned}
& X_{\partial}=\partial^{-1}(\theta) \\
& \chi_{\partial}^{*}\left(\mathfrak{D}_{\partial}, \pi\right)=\chi^{*}\left(\mathfrak{D}_{\partial} \cup \partial, \pi\right) \quad{ }^{\forall} \mathfrak{D}_{\partial} \in \mathfrak{D}_{X_{\partial}}
\end{aligned}
$$

where $D_{\partial} \cup \partial$ is a division on $X$ given by

$$
\left(D_{\partial} \cup \partial\right)^{-1}(\pi)=D_{\partial}^{-1}(\pi) \cup \partial^{-1}(\pi) \text {. }
$$

Let $X$ be a finite set. A judge $\chi^{*}$ on $X$ is said to be regular if

$$
\mathfrak{D}_{1}^{-1}(\pi) \supset \mathfrak{D}_{2}^{-1}(\pi) \text { implies } \chi^{*}\left(\mathfrak{D}_{1}, \pi\right) \geqq \chi^{*}\left(\mathfrak{D}_{2}, \pi\right),
$$

misère if

$$
\mathfrak{D}_{1}^{-1}(\pi) \supset \mathfrak{D}_{2}^{-1}(\pi) \quad \text { implies } \quad \chi^{*}\left(\mathfrak{D}_{1}, \pi\right) \leqq \chi^{*}\left(\mathfrak{D}_{2}, \pi\right)
$$

and trivial if $\chi^{*}$ is regular and also misère. A judge $\chi^{*}$ is trivial if and only if $\chi^{*} i_{i \mathfrak{D}_{X}}$ is constant for each player $\pi$.

A division space $\mathscr{D}^{*}=\left(X, \chi^{*}\right)$ is said to be regular, misère or trivial if $\chi^{*}$ is so, and denoted as $\mathscr{D}^{+}=\left(X, \chi^{+}\right), \mathscr{D}^{-}=\left(X, \chi^{-}\right)$or $\mathscr{D}^{0}=\left(X, \chi^{0}\right)$, respectively. A subset $S$ of $X$ is said to be negligible if $\mathscr{D}_{\partial}^{*}$ is trivial for each position $\partial$ over $X$ such that

$$
\partial^{-1}(\theta) \subset S
$$

Let $\mathscr{D}^{+}=\left(X, \chi^{+}\right)$be a regular division space and $\pi$ a player. Then a division $\mathfrak{D}$ over $X$ is called a $\pi$-division if $\chi^{+}(\mathfrak{D}, \pi)$ is 1 . A $\pi$-division $\mathfrak{D}_{0}$ over $X$ is said to be minimal if

$$
\chi^{+}(\mathfrak{D}, \pi)=1 \text { implies } \mathfrak{D}=\mathrm{D}_{0}
$$


for any division $\mathfrak{D}$ over $X$ such that

$$
\delta^{-1}(\pi) \subset \delta_{0}^{-1}(\pi)
$$

We put

$$
X^{0}=\{x \in X \mid\{x\} \text { is negligible }\}
$$

and call it the negligible part of $\mathscr{D}^{+} . \quad X^{0}$ is the sole maximal negligible subset of $X$. We put also

$$
X^{\varepsilon}=X-X^{0}
$$

and call it the essential part of $\mathscr{D}^{+}$.

Let $\mathscr{D}_{i}^{*}=\left(X_{i}, \chi_{i}^{*}\right)_{i=1,2}$ be division spaces. A pair $f=\left(f^{X}, \operatorname{sgn} f\right)$ of a mapping $f^{X}$ from $X_{1}$ to $X_{2}$ and a permutation $\operatorname{sgn} f$ of $\Pi$ is called a pseudo-homomorphism from $\mathscr{D}_{1}^{*}$ to $\mathscr{D}_{2}^{*}$ if the diagram below commutes:

$$
f^{\mathcal{D}} \times \operatorname{sgn} f: \quad \mathfrak{D}_{X_{1}} \times \Pi \longleftarrow \mathfrak{D}_{X_{2}} \times \Pi
$$

where $f^{D}$ is given by

$$
f^{D}\left(D_{2}\right)=\operatorname{sgn} f \circ D_{2} \circ f^{X} .
$$

A pseudo-homomorphism is called a pseudo-immersion, pseudo-contraction or pseudo-isomorphism when $f^{X}$ is injective, surjective or bijective. A pseudoisomorphism is called a pseudo-automorphism when $\mathscr{D}_{1}^{*}$ coincides with $\mathscr{D}_{2}^{*}$. The prefix "pseudo-" is omitted if $\operatorname{sgn} f=i d \Pi$ and replaced by "anti-" if $\operatorname{sgn} f=^{\wedge}$.

Let $\mathscr{D}^{*}$ be a division space. Then the set $\mathscr{A}_{u} \mathscr{D}^{*}$ consisting of pseudoautomorphisms of $\mathscr{D}^{*}$ naturally forms a group and its subset $\mathscr{A}_{n} \mathscr{D}^{*}$ consisting of automorphisms of $\mathscr{D}^{*}$ forms a normal subgroup of $\mathscr{A}_{u} \mathscr{D}^{*}$ of index 1 or 2. The division space $\mathscr{D}^{*}$ is said to be impartial if the above index is 2 and strongly impartial if $\left(i d_{X},{ }^{\wedge}\right)$ is an anti-automorphism.

We prepare several conventions on graphs. A graph is a pair $(X, \varphi)$ of a finite set $X$ and a mapping $\varphi$ from $X \times X$ to $\{0,1\}$ such that

i) $\varphi(x, x)=0 \quad \forall x \in X$

ii) $\varphi(y, x)=\varphi(x, y) \quad \forall x, y \in X$.

Let $(X, \varphi)$ be a graph. Then an element of $X$ is called a point. A point $x$ is said to be adjacent to a point $y$ if $\varphi(x, y)=1$. An edge is defined as an undirected 
pair of points adjacent to each other. For any point $x$ of $X$, we define the degree $e$ of $x$ by the number of points adjacent to $x$. A point $x$ is said to be ramified if $e \geqq 3$ and terminal if $e \leqq 1$. A terminal point is said to be isolated if $e=0$. The graph $(X, \varphi)$ is said to be discrete if $\varphi=0$, complete if $\varphi(x, y)=1$ for $x \neq y$ and connected if

$$
{ }^{3} x \in \mathfrak{D}^{-1}(T),{ }^{\exists} y \in \mathfrak{D}^{-1}(\perp) \quad \varphi(x, y)=1
$$

for any non-constant division $\mathfrak{D}$ on $X$. The graph $(X, \varphi)$ is said to be cyclic if it is connected and the degree of $x$ is 2 for any point $x$. Let $Y$ be a subset of $X$. Then we have a subgraph $\left(Y,\left.\varphi\right|_{Y \times Y}\right)$, where $\left.\varphi\right|_{Y \times Y}$ is often omitted. $Y$ is said to be discrete, complete, connected or cyclic if $\left(Y,\left.\varphi\right|_{Y \times Y}\right)$ is so. Let $n \geqq 1$ and $\left(x_{0}, x_{n}\right)$ be a pair of points of $X$. Then a series $x_{1}, \ldots, x_{n-1}$ in $Y$ is called a chain in $Y$ from $x_{0}$ to $x_{n}$ if

$$
\varphi\left(x_{i-1}, x_{i}\right)=1 \quad 0<i \leqq n .
$$

A series of length 0 is regarded as a chain from a point to one adjacent to it. The graph $(X, \varphi)$ is connected if and only if there is a chain in $X$ from any point of $X$ to any other point of it.

\section{§2. G-Connex}

Let $G$ be a finite group. Then we denote by $\mathscr{S}_{w b}(G)$ the set consisting of the subgroups of $G$ and by $\&$ the family consisting of subsets $g$ of $\mathscr{S}_{u} b(G)$ such that

$$
\mathfrak{g} \ni H, H \supset H^{\prime} \text { implies } \mathfrak{g} \ni H^{\prime} .
$$

Let $\tilde{X}$ be a finite set admitting an action of $G$. Then we denote by $X=G \backslash \tilde{X}$ the orbit space and denote by $G \backslash$ the natural surjection from $\tilde{X}$ to $X$.

We fix a finite group $G$ in the rest of this paper. A pair $(\tilde{X}, \Phi)$ of a finite set $\tilde{X}$ admitting an action of $G$ and a mapping $\Phi$ from $\tilde{X} \times \tilde{X} \times \Pi$ to $\{0,1\}$ is called a $\boldsymbol{G}$-bigraph if

$$
\Phi(g(\tilde{x}), g(\tilde{y}), \pi)=\Phi(\tilde{x}, \tilde{y}, \pi) \quad{ }^{\forall} g \in G
$$

and $\left(\tilde{X}, \Phi_{\circ} \cdot \frac{\pi}{\tilde{X} \times \bar{X}}\right)$ is a graph for each player $\pi$. From now on we denote $\Phi_{\circ} \cdot \frac{\pi}{\tilde{X} \times \tilde{X}}$ by $\varphi^{\pi}$.

Let $(\tilde{X}, \Phi)$ be a $G$-bigraph, $\pi$ a player and $\partial$ a position on $X$. Then we define the stabilizer ideal $\mathfrak{g}[\partial, \pi]$ of $(\partial, \pi)$ by 


$$
\mathfrak{g}[\partial, \pi] \ni H
$$

iff ${ }^{3} A$ : non-empty $H$-invariant $\varphi^{\pi}$-connected subset of $(\partial \circ G \mid)^{-1}(\pi)$.

We define also another $G$-bigraph $\left(\tilde{X}_{\partial}, \Phi_{\partial}\right)$ as follows:

$$
\tilde{X}_{\partial}=(\partial \circ G \backslash)^{-1}(\theta)
$$

where $G$ acts on it as on a $G$-invariant subset of $\tilde{X}$,

$$
\Phi_{\partial}(\tilde{x}, \tilde{y}, \pi)=1
$$

iff $\tilde{x}$ and $\tilde{y}$ are distinct and they are in the same $\varphi^{\pi}$-connected component of $(\partial \circ G \backslash)^{-1}(\pi) \cup\{\tilde{x}, \tilde{y}\}$.

Lemma 2-1. Let $(\tilde{X}, \Phi)$ be a G-bigraph, $\partial$ a position on $X$ and $\mathfrak{D}_{\partial} a$ division on $X_{\partial}$. Then

$$
\mathfrak{g}\left[\mathfrak{D}_{\partial} \cup \partial, \pi\right]=\mathfrak{g}_{\partial}\left[\mathfrak{D}_{\partial}, \pi\right] \cup \mathfrak{g}[\partial, \pi],
$$

where $\mathrm{g}_{\partial}\left[\mathrm{D}_{\partial}, \pi\right]$ is the stabilizer ideal of $\left(D_{\partial}, \pi\right)$ with respect to the G-bigraph $\left(\tilde{X}_{\partial}, \Phi_{\partial}\right)$.

Proof.

$$
H \in \mathfrak{g}\left[\partial_{\partial} \cup \partial, \pi\right]
$$

$\Leftrightarrow{ }^{\exists} A$ : non-empty $H$-invariant $\varphi^{\pi}$-connected subset of

$$
\left(\left(\mathfrak{D}_{\partial} \cup \partial\right) \circ G \backslash\right)^{-1}(\pi)
$$

$$
\begin{aligned}
& \Leftrightarrow\left\{\begin{array}{c}
{ }^{\exists} A_{\hat{\imath}}: \text { non-empty } H \text {-invariant } \varphi_{\partial}^{\pi} \text {-connected subset of } \\
\left(\mathfrak{D}_{\partial} \circ G \backslash\right)^{-1}(\pi) \\
\text { or }{ }^{\exists} A: \text { non-empty } H \text {-invariant } \varphi^{\pi} \text {-connected subset of } \\
\left(\partial_{\circ} G \backslash\right)^{-1}(\pi)
\end{array}\right. \\
& \Leftrightarrow H \in \mathfrak{g}_{\partial}\left[\mathfrak{D}_{\partial}, \pi\right] \cup \mathfrak{g}[\partial, \pi] .
\end{aligned}
$$

Lemma 2-2. Let $(\tilde{X}, \Phi)$ be a G-bigraph, $\pi$ a player and $\grave{D}_{1}$ and $\delta_{2}$ divisions on $X$ such that

$$
D_{1}^{-1}(\pi) \supset D_{2}^{-1}(\pi)
$$

Then

$$
\mathfrak{g}\left[\mathrm{D}_{1}, \pi\right] \supset \mathfrak{g}\left[\mathfrak{D}_{2}, \pi\right] \text {. }
$$

Proof. We define a position $\partial$ on $X$ and a division $D_{\partial}$ on $X_{\partial}$ as follows:

$$
\partial^{-1}(\pi)=\mathfrak{D}_{2}^{-1}(\pi), \quad \partial^{-1}(\hat{\pi})=\mathfrak{D}_{1}^{-1}(\hat{\pi}), \quad \grave{D}_{\partial}\left(x_{\partial}\right)=\pi .
$$


Then we have by the previous lemma,

$$
\mathfrak{g}\left[\mathfrak{D}_{1}, \pi\right]=\mathfrak{g}\left[\partial \cup \mathfrak{D}_{\partial}, \pi\right] \supset \mathfrak{g}[\partial, \pi]=\mathfrak{g}\left[\mathfrak{D}_{2}, \pi\right],
$$

which completes our proof.

A triplet $\mathscr{C}=\left(\tilde{X}, \Phi, \chi^{+}\right)$of a finite set $\tilde{X}$ admitting a group action of $G$, a mapping $\Phi$ and a regular judge $\chi^{+}$on $X$ is called a $G$-connex if $(\tilde{X}, \Phi)$ is a $G$ bigraph and

$$
\mathfrak{g}[\mathfrak{D}, \pi] \supset \mathfrak{g}\left[\mathfrak{D}^{\prime}, \pi\right] \text { implies } \chi^{+}(\mathbb{D}, \pi) \geqq \chi^{+}\left(\mathfrak{D}^{\prime}, \pi\right) .
$$

Let $\mathscr{C}=\left(\tilde{X}, \Phi, \chi^{+}\right)$be a $G$-connex. Then we denote the division space $\left(X, \chi^{+}\right)$by $\mathscr{C}^{+}$. We put

$$
\begin{aligned}
& \tilde{X}^{\varepsilon}=(G \backslash)^{-1}\left(X^{\varepsilon}\right) \\
& \tilde{X}^{0}=(G \backslash)^{-1}\left(X^{0}\right)
\end{aligned}
$$

and call an element of them an essential point and a negligible point of $\mathscr{C}$ respectively. These notion will be fixed throughout this paper. $\mathrm{A} \boldsymbol{Z} / 2 \boldsymbol{Z}$-connex is simply called a connex.

Lemma 2-3. Assume that $G$ is of prime order. Let $\mathscr{C}=\left(\tilde{X}, \Phi, \chi^{+}\right)$be a G-connex and $\pi$ a player. Suppose that there exists a $\pi$-division $\mathrm{D}_{0}$ such that

$$
\mathrm{g}\left[\mathrm{D}_{0}, \pi\right] \not \supset G .
$$

Then $\chi^{+}(\mathrm{D}, \pi)=1$ for any division $\mathrm{D}$ on $X$ or

$$
\chi^{+}(\mathfrak{D}, \pi)=1 \quad \text { iff } \quad \mathrm{g}[\mathrm{D}, \pi] \neq \emptyset .
$$

This lemma is easily verified and its proof is omitted.

Lemma 2-4. Assume $|G|=2$. Let $\mathscr{C}=\left(\tilde{X}, \Phi, \chi^{+}\right)$be a connex, $\pi$ a player and $\grave{D}_{0}$ a minimal $\pi$-division on $X$. Suppose

$$
\chi^{+}(\mathfrak{D}, \pi)=1 \quad \text { iff } \quad \mathfrak{g}[\mathfrak{D}, \pi] \ni G .
$$

We put $\tilde{A}=\left(\mathfrak{D}_{0} \circ G \backslash\right)^{-1}(\pi)$. Then one of the following two conditions holds:

i) $\left|\mathfrak{D}_{0}^{-1}(\pi)\right|=1$ and $\left(A,\left.\varphi^{\pi}\right|_{\tilde{A} \times \tilde{A}}\right)$ is a connected graph,

ii) $\left|\mathfrak{D}_{0}^{-1}(\pi)\right| \geqq 2$, the action of $G$ is fixed-point-free on $\tilde{A}$ and $\left(\tilde{A},\left.\varphi^{\pi}\right|_{\tilde{A} \times \tilde{A}}\right)$ is a cyclic graph.

Proof. We easily have $\left|\mathfrak{b}_{0}^{-1}(\pi)\right| \geqq 1$. First suppose $\left|\mathfrak{b}_{0}^{-1}(\pi)\right|=1$. Then $\left(\tilde{A},\left.\varphi^{\pi}\right|_{\tilde{A} \times \tilde{A}}\right)$ must be a connected graph of one or two points. Next suppose $\left|D_{0}^{-1}(\pi)\right| \geqq 2$. We easily see that the action of $G$ is free on $\tilde{A}$, because of the 
minimality of $\mathfrak{d}_{0}$. We can find a series $\tilde{x}=\tilde{x}_{0}, \tilde{x}_{1}, \ldots, \tilde{x}_{m}=\alpha(\tilde{x})$ in $\tilde{A}$ satisfying

$$
\varphi^{\pi}\left(\tilde{x}_{i-1}, \tilde{x}_{i}\right)=1 \quad 1 \leqq{ }_{i} \leqq n
$$

with minimal length $m$ where $\alpha$ is the generator of $G$. Since $\mathfrak{D}_{0}$ is a minimal $\pi$-division, $G \backslash\left(\tilde{x}_{i}\right)$ is distinct from $G \backslash\left(\tilde{x}_{j}\right)$ for $0<i<j \leqq m$. Therefore $\tilde{A}=\cup_{i=0}^{m-1} G$. $\left\{x_{i}\right\}$ and $\left(\tilde{A},\left.\varphi^{\pi}\right|_{\tilde{A} \times \tilde{A}}\right)$ must be a cyclic graph, because of the minimality of $D_{0}$ (see Figure 1).

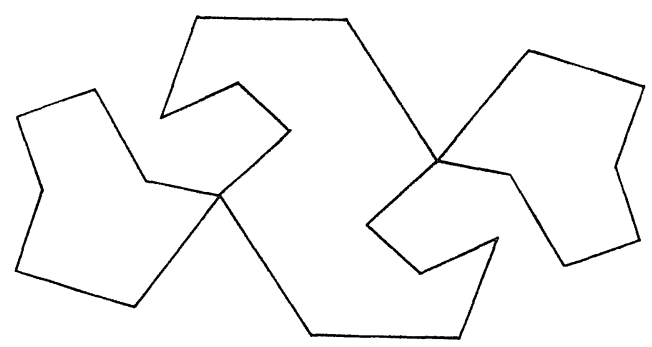

Figure 1.

Now our proof is completed.

Theorem 1. Let $\mathscr{C}=\left(\tilde{X}, \Phi, \chi^{+}\right)$be a G-connex and $\partial$ a position over $X$. We define a triplet $\mathscr{C}_{\partial}=\left(\tilde{X}_{\partial}, \Phi_{\partial}, \chi_{\partial}^{+}\right)$as follows:

$$
\begin{aligned}
& \left(\tilde{X}_{\partial}, \Phi_{\partial}\right) \text { is the G-bigraph introduced from }(\tilde{X}, \Phi, \partial) \\
& \chi_{\partial}^{+}\left(\mathfrak{D}_{\partial}, \pi\right)=\chi^{+}\left(\mathfrak{D}_{\partial} \cup \partial, \pi\right) \text { for a division } \mathfrak{D}_{\partial} \text { on } X_{\partial} \text {. }
\end{aligned}
$$

Then $\mathscr{C}_{\partial}$ is a G-connex.

Proof. We have to prove only

$$
\mathfrak{g}_{\partial}\left[\mathfrak{D}_{\partial}^{\prime}, \pi\right] \supset \mathfrak{g}_{\partial}\left[\mathfrak{D}_{\partial}, \pi\right] \text { implies } \chi_{\hat{\partial}}^{+}\left(\mathfrak{D}_{\partial}^{\prime}, \pi\right) \geqq \chi_{\partial}^{+}\left(\mathfrak{D}_{\partial}, \pi\right) \text {. }
$$

Let $\mathrm{D}_{\partial}^{\prime}$ and $\mathrm{D}_{\partial}$ be divisions on $X_{\partial}$ such that

$$
\mathfrak{g}_{\partial}\left[\mathfrak{b}_{\partial}^{\prime}, \pi\right] \supset \mathfrak{g}_{\partial}\left[\mathfrak{D}_{\partial}, \pi\right] \text {. }
$$

Then by Lemma $2-1$,

$$
\begin{aligned}
& \mathfrak{g}\left[\mathfrak{D}_{\partial}^{\prime} \cup \partial, \pi\right]=\mathfrak{g}_{\partial}\left[\mathfrak{D}_{\partial}^{\prime}, \pi\right] \cup \mathfrak{g}[\partial, \pi] \\
& \quad \supseteq \mathfrak{g}_{\partial}\left[\mathfrak{D}_{\partial}, \pi\right] \cup \mathfrak{g}[\partial, \pi]=\mathfrak{g}\left[\mathfrak{D}_{\partial} \cup \partial, \pi\right] .
\end{aligned}
$$

Therefore

$$
\begin{aligned}
& \chi_{\partial}^{+}\left(D_{\partial}^{\prime}, \pi\right)=\chi^{+}\left(D_{\partial}^{\prime} \cup \partial, \pi\right) \\
& \quad \geqq \chi^{+}\left(D_{\partial} \cup \partial, \pi\right)=\chi_{\partial}^{+}\left(D_{\partial}, \pi\right) .
\end{aligned}
$$

Now our theorem is verified. 


\section{§3. Homomorphisms}

Let $\mathscr{C}_{i}=\left(\tilde{X}_{i}, \Phi_{i}, \chi_{i}^{+}\right)_{i=1,2}$ be $G$-connexes. Then a triplet $f=\left(f^{G}, f^{\tilde{X}}, \operatorname{sgn} f\right)$ of a group automorphism $f^{G}$ of $G$, a mapping $f^{\tilde{X}}$ from $\tilde{X}_{1}$ to $\tilde{X}_{2}$ and a permutation $\operatorname{sgn} f$ of $\Pi$ is called a pseudo-homomorphism if the following conditions hold :

i) the following diagram commutes where ( ) denotes the group action,

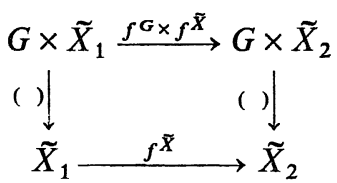

ii) if $\tilde{A} \subset \tilde{X}_{1}$ is $\varphi_{1}^{\pi}$-connected, then $f^{\tilde{X}}(\tilde{A}) \subset \tilde{X}_{2}$ is $\varphi_{2}^{\mathrm{sgn} f(\pi)}$-connected,

iii) $\left(f^{X}, \operatorname{sgn} f\right)$ is a pseudo-homomorphism from $\mathscr{C}_{1}^{+}$to $\mathscr{C}_{2}^{+}$where

$$
f^{X} \circ G \backslash=G \backslash f^{\bar{X}} .
$$

A pseudo-homomorphism $f=\left(f^{G}, f^{\bar{x}}, \operatorname{sgn} f\right)$ is called a pseudo-immersion if $f^{\tilde{X}}$ is injective and is called a pseudo-contraction if $f^{\tilde{x}}$ is surjective and

$$
\begin{array}{r}
\Phi_{2}\left(\tilde{x}_{2}, \tilde{y}_{2}, \pi\right)=1 \text { implies }{ }^{\exists} \tilde{x}_{1} \in f^{\bar{x}}\left(\tilde{x}_{2}\right),{ }^{3} \tilde{y}_{1} \in f^{\tilde{x}}\left(\tilde{y}_{2}\right) \\
\Phi_{1}\left(\tilde{x}_{1}, \tilde{y}_{1}, \operatorname{sgn} f(\pi)\right)=1 .
\end{array}
$$

A pseudo-homomorphism $f$ is called a pseudo-isomorphism if it is a pseudoimmersion and also a pseudo-contraction. A pseudo-isomorphism from $\mathscr{C}_{1}$ to $\mathscr{C}_{1}$ is called a pseudo-automorphism of $\mathscr{C}_{1}$. The prefix "pseudo-" is omitted if $\operatorname{sgn} f=i d_{\pi}$ and replaced by "anti- if $\operatorname{sgn} f=$ ".

Let $\mathscr{C}$ be a $G$-connex. Then the set $\mathscr{A} u \mathscr{C} \mathscr{C}$ of pseudo-automorphisms of $\mathscr{C}$ naturally admits a group structure. The subset $\mathscr{A}_{u} \boldsymbol{t}_{\Pi} \mathscr{C}$ of automorphisms of $\mathscr{A}_{u t \mathscr{C}}$ is a normal subgroup of $\mathscr{A}_{u t \mathscr{C}}$ of index 1 or 2 . The $G$-connex $\mathscr{C}$ is said to be impartial if the above index is 2 and strongly impartial if $\left(i d_{G}, i d_{\tilde{X}},{ }^{\wedge}\right)$ is an anti-automorphism of $\mathscr{C}$. As is easily seen $\mathscr{C}$ is strongly impartial if $\left(\sigma, i d_{\tilde{X}},{ }^{\wedge}\right)$ is an anti-automorphism for a group automorphism $\sigma$ of $G$.

Proposition 1. Assume that $G$ is of prime order. Let $\mathscr{C}_{i}=\left(\tilde{X}_{i}, \Phi_{i}\right.$, $\left.\chi_{i}^{+}\right)_{i=1,2}$ be G-connexes and $f=\left(f^{G}, f^{\tilde{X}}, i d_{\pi}\right)$ a homomorphism from $\mathscr{C}_{1}$ to $\mathscr{C}_{2}$. Then there exists a sole triplet $\left(\mathscr{C}, f_{1}, f_{2}\right)$, up to isomorphism, of a G-connex $\mathscr{C}$, a contraction $f_{1}$ from $\mathscr{C}_{1}$ to $\mathscr{C}$ and an immersion $f_{2}$ from $\mathscr{C}$ to $\mathscr{C}_{2}$ such that $f=f_{2} \circ f_{1}$. 
Proof. We put

$$
\begin{aligned}
& \tilde{X}=\operatorname{Im} f^{\tilde{X}}, \\
& \begin{array}{l}
\Phi(\tilde{x}, \tilde{y}, \pi)=1 \quad \text { iff } \quad \tilde{x} \neq \tilde{y} \text { and } \Phi_{1}\left(\tilde{x}_{1}, \tilde{y}_{1}, \pi\right)=1 \\
\text { for a pair }\left(\tilde{x}_{1}, \tilde{y}_{1}\right) \in f^{\tilde{x}^{-1}}(\tilde{x}) \times f^{\tilde{X}^{-1}}(\tilde{y}), \\
\chi^{+}(\mathfrak{D}, \pi)=\chi_{2}^{+}\left(\mathfrak{D}_{2}, \pi\right)
\end{array}
\end{aligned}
$$

where $\mathfrak{D}_{2} \circ f_{2}^{X}=\mathfrak{D}$, to construct $\mathscr{C}=\left(\tilde{X}, \Phi, \chi^{+}\right)$. Here $\tilde{X}$ is $G$-stable, since $f^{G}$ is surjective. We put also

$$
\begin{aligned}
& f_{1}^{\tilde{X}}\left(\tilde{x}_{1}\right)=f^{\tilde{X}}\left(\tilde{x}_{1}\right) \\
& f_{2}^{\tilde{X}}(\tilde{x})=\tilde{x}
\end{aligned}
$$

to construct $f_{1}=\left(f^{G}, f_{1}^{\bar{X}}, i d_{\Pi}\right)$ and $f_{2}=\left(i d_{G}, f_{2}^{\bar{X}}, i d_{\Pi}\right)$. It is easy to see that there exists no other desired triplet $\left(\mathscr{C}, f_{1}, f_{2}\right)$, because $\operatorname{Im} f_{2}^{\bar{X}}$ must coincide with $\operatorname{Im} f^{\tilde{x}}$. Now we have to prove only

$$
\mathfrak{g}[\mathfrak{D}, \pi] \supset \mathfrak{g}\left[\mathfrak{D}^{\prime}, \pi\right] \quad \text { implies } \quad \chi^{+}(\mathfrak{D}, \pi) \geqq \chi^{+}\left(\mathfrak{D}^{\prime}, \pi\right) .
$$

Let $\mathfrak{D}$ and $\mathfrak{D}^{\prime}$ be divisions on $X$ such that $g[D, \pi] \supset g\left[\mathfrak{D}^{\prime}, \pi\right]$. Then we fix divisions $\mathfrak{D}_{2}$ and $\mathfrak{D}_{2}^{\prime}$ on $X$ such that $\mathfrak{D}_{2} \circ f_{2}^{X}=\mathfrak{D}$ and $\mathfrak{D}_{2}^{\prime} \circ f_{2}^{X}=\mathfrak{D}^{\prime}$. First assume

$$
\mathrm{g}\left[\mathfrak{D}^{\prime}, \pi\right] \not \nexists G \text {. }
$$

Then we easily obtain

$$
\mathfrak{g}_{1}\left[\mathrm{D} \circ f_{1}^{X}, \pi\right] \supset \mathfrak{g}_{1}\left[\mathrm{D}^{\prime} \circ f_{1}^{X}, \pi\right]
$$

which implies

$$
\begin{aligned}
& \chi^{+}(\mathfrak{D}, \pi)=\chi_{2}^{+}\left(\mathfrak{D}_{2}, \pi\right)=\chi_{1}^{+}\left(\mathfrak{D}_{2} \circ f^{X}, \pi\right)=\chi_{1}^{+}\left(\mathfrak{D}_{1}^{X}, \pi\right) \\
& \quad \geqq \chi_{1}^{+}\left(\mathfrak{D}^{\prime} \circ f_{1}^{X}, \pi\right)=\chi_{1}^{+}\left(\mathfrak{D}_{2}^{\prime} \circ f^{X}, \pi\right)=\chi_{2}^{+}\left(\mathfrak{D}_{2}^{\prime}, \pi\right)=\chi^{+}\left(\mathfrak{D}^{\prime}, \pi\right) .
\end{aligned}
$$

Next assume

$$
\mathfrak{g}\left[\mathfrak{D}^{\prime}, \pi\right] \ni G
$$

Then we have

$$
\mathfrak{g}_{2}\left[\mathfrak{D}_{2}, \pi\right] \supset \mathfrak{g}[\mathfrak{D}, \pi] \supset \mathfrak{g}\left[\mathfrak{D}^{\prime}, \pi\right] \ni G
$$

which implies

$$
\mathfrak{g}_{2}\left[\mathfrak{D}_{2}, \pi\right] \supset \mathfrak{g}_{2}\left[\mathfrak{D}_{2}^{\prime}, \pi\right]
$$

Now we have

$$
\chi^{+}(\mathrm{D}, \pi)=\chi_{2}^{+}\left(\mathrm{D}_{2}, \pi\right) \geqq \chi_{2}^{+}\left(\mathrm{D}_{2}^{\prime}, \pi\right)=\chi^{+}\left(\mathrm{D}^{\prime}, \pi\right),
$$

which completes our proof. 
A $G$-connex $\mathscr{C}$ is said to be primitive if any immersion to $\mathscr{C}$ is an isomorphism. If $|G|=2$, then the automorphism of $G$ is trivial, and we omit $f^{G}$ of a pseudo-homomorphism $\left(f^{G}, f^{\bar{x}}, \operatorname{sgn} f\right)$ of connexes.

Theorem 2. Assume $|G|=2$. Let $\mathscr{C}=\left(\tilde{X}, \Phi, \chi^{+}\right)$be a connex. Then there exists a sole pair $\left(\mathscr{C}_{0}, f\right)$, up to isomorphisms, of a primitive connex $\mathscr{C}_{0}=\left(\tilde{X}_{0}, \Phi_{0}, \chi_{0}^{+}\right)$and an immersion $f=\left(f^{\tilde{x}}, \operatorname{sgn} f\right)$ from $\mathscr{C}_{0}$ to $\mathscr{C}$.

Proof. We put

$$
\begin{aligned}
& \tilde{X}_{0}=\tilde{X}^{\varepsilon} \\
& \chi_{0}^{+}\left(\mathfrak{D}_{0}, \pi\right)=\chi^{+}(\mathcal{D}, \pi)
\end{aligned}
$$

where $\mathfrak{b}_{0}=\left.\mathfrak{d}\right|_{X_{0}}$. Let $\pi$ be a player. First assume that there exists a $\pi$-division D on $X$ such that

$$
\mathrm{g}[\mathrm{D}, \pi] \not \supset G
$$

Then we put

$$
\Phi_{0}\left(\tilde{x}_{0}, \tilde{y}_{0}, \pi\right)=0 \quad \forall \tilde{x}_{0}, \tilde{y}_{0} \in \tilde{X}_{0} .
$$

Next assume that every $\pi$-division satisfies

$$
g[d, \pi] \ni G .
$$

Then we put

$$
\Phi_{0}\left(\tilde{x}_{0}, \tilde{y}_{0}, \pi\right)=1
$$

iff $\Phi\left(\tilde{x}_{0}, \tilde{y}_{0}, \pi\right)=1$ and $\left(\mathrm{D}_{0} \circ G \backslash\right)\left(\tilde{x}_{0}\right)=\left(\mathfrak{D}_{0} \circ G \backslash\right)\left(\tilde{y}_{0}\right)=\pi$ for a minimal $\pi$-division $\mathfrak{D}_{0}$ over $X$.

We put

$$
f^{\bar{x}}\left(\tilde{x}_{0}\right)=\tilde{x}_{0} .
$$

Now by Lemma $2-3$ and Lemma $2-4, \mathscr{C}_{0}=\left(\tilde{X}_{0}, \Phi_{0}, \chi_{0}^{+}\right)$is a primitive connex and $\left(f^{\tilde{x}}, i d_{\Pi}\right)$ is an immersion from $\mathscr{C}_{0}$ to $\mathscr{C}$. The uniqueness follows from Proposition 1 immediately.

\section{§4. Mass Spaces and Their Connexes}

A strongly impartial connex $\mathscr{C}=\left(\tilde{X}, \Phi, \chi^{+}\right)$is said to be tame if

$$
\chi^{+}(\mathfrak{D}, \pi)=1 \quad \text { iff } \quad \mathfrak{g}[\mathrm{d}, \pi] \ni G .
$$

By Lemma 2-3, there exists a sole strongly impartial connex $\mathscr{C}=\left(\tilde{X}, \Phi, \chi^{+}\right)$ 
which is not tame, where $\tilde{X}$ is a set of two points with a nontrivial action of $G$, $\Phi=0$ and

$$
\chi^{+}(\mathfrak{D}, \pi)=1 \quad \text { iff } \quad \mathrm{g}[\mathrm{D}, \pi] \neq \varnothing .
$$

In the rest of this paper, we deal with tame connexes only. We assume that $G$ is a cyclic group of order 2 and denote the generator of $G$ by $\alpha$. For any tame connex $\mathscr{C}=\left(\tilde{X}, \Phi, \chi^{+}\right)$, we define a graph $(\tilde{X}, \varphi)$ by $\varphi=\varphi^{\top}=\varphi^{\perp}$. From now on, we identify a tame connex with a graph $(\tilde{X}, \varphi)$ admitting a group action of $G$ such that the following $\chi^{+}$is a judge on $X$ :

$$
\chi^{+}(\mathfrak{D}, \pi)=1 \quad \text { iff } \quad \mathfrak{g}[\mathfrak{D}, \pi] \ni G .
$$

We identify also a homomorphism $f=\left(f^{\tilde{X}}, i d_{\Pi}\right)$ with $f^{\tilde{X}}$ while there is no confusion.

Let $\mathscr{C}=(\tilde{X}, \varphi)$ be a tame connex and $\tilde{F}$ a $G$-invariant subset of $\tilde{X}$. For any connected subset $\tilde{C}$ of $\tilde{X}-\tilde{F}$, we define the support $\operatorname{Supp}_{\tilde{F}}(\widetilde{C})$ of $\tilde{C}$ with respect to the frame $\widetilde{F}$ by

$$
\operatorname{Supp}_{\tilde{F}}(\widetilde{C})=\left\{\tilde{f} \in \tilde{F}|\varphi|_{\tilde{f} \times \tilde{C}_{0}} \neq 0\right\}
$$

where $\widetilde{C}_{0}$ is the connected component containing $\tilde{C}$ in $\tilde{X}-\tilde{F}$. For any subset $\tilde{A}$ of $\tilde{F}$, we define the web $\mathrm{Web}_{\tilde{F}}(\tilde{A})$ of $\tilde{A}$ by

$$
\mathrm{Web}_{\tilde{F}}(\tilde{A})=\left\{\tilde{x} \in \tilde{X}-\tilde{F} \mid \operatorname{Supp}_{\tilde{F}}(\{\tilde{x}\}) \subset \tilde{A}\right\},
$$

and denote $\operatorname{Web}_{\tilde{F}}(\tilde{A}) \cup \tilde{A}$ by $\bar{W}_{\tilde{F}}(\tilde{A})$.

Lemma 4-1. Let $\mathscr{C}=(\tilde{X}, \varphi)$ be a tame connex, $\tilde{F}$ a G-invariant subset of $\tilde{X}$ and $\widetilde{C}$ a connected subset of $\tilde{X}-\widetilde{F}$. Assume that $\operatorname{Supp}_{\widetilde{F}}(\widetilde{C})$ is a non-empty complete subset of $\widetilde{F}$. Then $\widetilde{C}$ is negligible.

Proof. We have to show our assertion only in case where $\tilde{C}$ is a connected component. Suppose that there exists an essential point $\tilde{x}_{0}$ of $\tilde{C}$ and a minimal $T$-division $\mathfrak{D}$ such that

$$
\mathrm{D} \circ G \backslash\left(\tilde{x}_{0}\right)=\top .
$$

Then we choose a chain $\tilde{x}_{1}, \ldots, \tilde{x}_{l-1}$ in $\tilde{X}$ from $\tilde{x}_{0}$ to $\alpha\left(\tilde{x}_{0}\right)$ satisfying $l=\left|D^{-1}(T)\right|$. Suppose that this chain is in $\tilde{C}$. Then $\operatorname{Supp}_{\tilde{F}}(\widetilde{C})$ is a non-empty G-invariant connected set since $\widetilde{C}$ is $G$-invariant. Therefore we have

$$
\mathfrak{g}\left[\mathfrak{D}^{\prime}, T\right]=\mathfrak{g}\left[\mathfrak{D}^{\prime}, \perp\right] \ni G
$$

for the division $\mathfrak{D}^{\prime}$ given by 


$$
\mathfrak{D}^{\prime}(\tilde{x})=\top \quad \text { iff } \quad \tilde{x} \in \tilde{C},
$$

a contradiction. Now the relevant chain is not in $\tilde{C}$. Let $j$ be the minimum and $k$ the maximum of the number $i$ such that $\tilde{x}_{i} \notin C$. Then we have

$$
\left\{\tilde{x}_{j}, \alpha\left(\tilde{x}_{k}\right)\right\} \subset \operatorname{Supp}_{\tilde{F}}(\tilde{C}) .
$$

By Lemma 2-4, $(\mathbb{D} \circ G \backslash)^{-1}(T)$ is cyclic. Then $\alpha\left(\tilde{x}_{k}\right) \neq \tilde{x}_{j}$. On the other hand $\alpha\left(\tilde{x}_{k}\right)$ is a point of $\operatorname{Supp}_{\tilde{F}}(\tilde{C})$. Then $\alpha\left(\tilde{x}_{k}\right)$ is adjacent to $\tilde{x}_{j}$ and $(\mathfrak{D} \circ G \backslash)^{-1}(T)$ is not cyclic. Now our lemma is verified (see Figure 2).

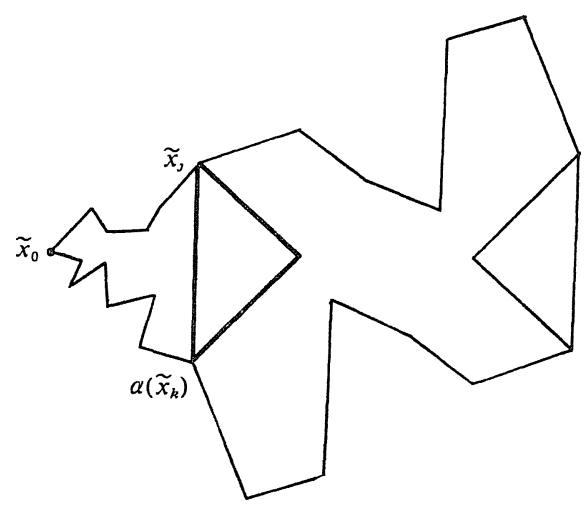

Figure 2.

Lemma 4-2. Let $\tilde{F}^{\prime} \subset \tilde{F} \subset \tilde{X}$ and $\tilde{C}$ a connected subset of $\tilde{X}-\tilde{F}$. Then we have

$$
\bar{W}_{\widetilde{F}^{\prime}}\left(\operatorname{Supp}_{\widetilde{F}^{\prime}}(\widetilde{C})\right) \supset \bar{W}_{\widetilde{F}}\left(\operatorname{Supp}_{\widetilde{F}}(\widetilde{C})\right) .
$$

Proof. Let $\tilde{y}_{0}$ be a point of $\bar{W}_{\tilde{F}}\left(\operatorname{Supp}_{\tilde{F}}(\widetilde{C})\right)$. We consider an arbitrary chain $\tilde{y}_{1}, \ldots, \tilde{y}_{n-1}$ in $\tilde{X}-\tilde{F}^{\prime}$ to a point $\tilde{y}_{n}$ of $\tilde{F}^{\prime}$. Then, we find the minimal number $k$ such that $\tilde{y}_{k} \in \tilde{F}$, which is a point of $\operatorname{Supp}_{\tilde{F}}(\tilde{C})$. Therefore we find a chain $\tilde{w}_{1}, \ldots, \tilde{w}_{m}$ in $\tilde{X}-\tilde{F}$ from a point $\tilde{w}_{0}$ of $\tilde{C}$ to $\tilde{y}_{k}$. Now $\tilde{w}_{1}, \ldots, \tilde{w}_{m}, \tilde{y}_{k}, \tilde{y}_{k+1}, \ldots$, $\tilde{y}_{n-1}$ is a chain in $\tilde{X}-\tilde{F}^{\prime}$ from $\tilde{w}_{0}$ to $\tilde{y}_{n}$ (see Figure 3 ). Hence

$$
\tilde{y}_{n} \in \operatorname{Supp}_{\tilde{F}^{\prime}}(\tilde{C}) \text {. }
$$




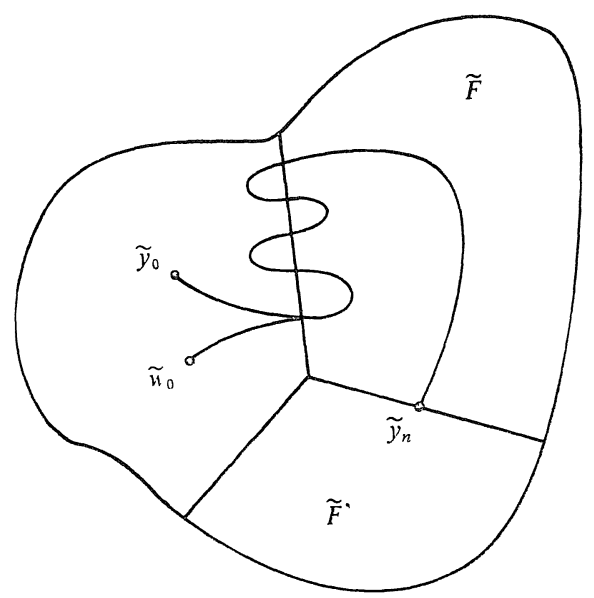

Figure 3.

Thus our lemma is verified.

Let $\mathscr{C}_{0}=\left(\tilde{X}_{0}, \varphi_{0}\right)$ be a tame connex and $f=\left(f^{\tilde{X}}, i d_{I I}\right)$ an immersion from $\mathscr{C}_{0}$ to $\mathscr{C}$. Then $f$ is called a $\nabla$-immersion, if $\varphi_{0}=\varphi \circ\left(f^{\tilde{X}} \times f^{\tilde{X}}\right)$ and $\operatorname{Supp}_{\tilde{F}}(\tilde{A})$ is complete with respect to $\varphi$ for a connected subset $\tilde{A}$ of $\tilde{X}-\tilde{F}$ where $\widetilde{F}=\operatorname{Im} f^{\tilde{X}}$.

We define a tame connex $\mathscr{M}=(\tilde{X}, \varphi)$ by $|\tilde{X}|=1$ and $\varphi=0$. Then we have the following theorem.

Theorem 3. Let $\mathscr{C}$ be a tame connex with a G-invariant point. Then $\mathscr{C}$ has a $\nabla$-immersion from $\mathscr{M}$.

The proof is easy and is omitted.

We define a series of division spaces. Let $m$ be a strictly positive integer. Then we define the mass space $\mathscr{M}_{2 m-1}^{+}=\left(X, \chi^{+}\right)$as follows:

$$
\begin{aligned}
& |X|=2 m-1 \\
& \chi^{+}(\mathfrak{D}, \pi)=1 \quad \text { iff } \quad\left|\mathfrak{D}^{-1}(\pi)\right| \geqq m .
\end{aligned}
$$

For $m=1,2,3$, we define tame connexes $\mathscr{M}_{2 m-1}=(\tilde{X}, \varphi)$ as follows (see Figure 4 and Figure 5):

$$
\begin{aligned}
& \tilde{X}=\left\{x_{i}, \alpha\left(x_{i}\right) \mid i=1, \ldots, 2 m-1\right\},
\end{aligned}
$$

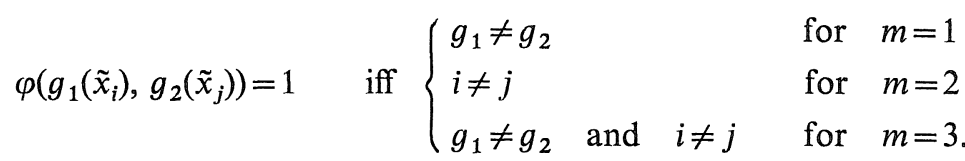




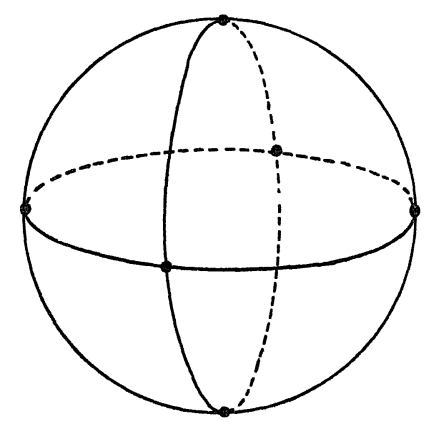

Figure 4.

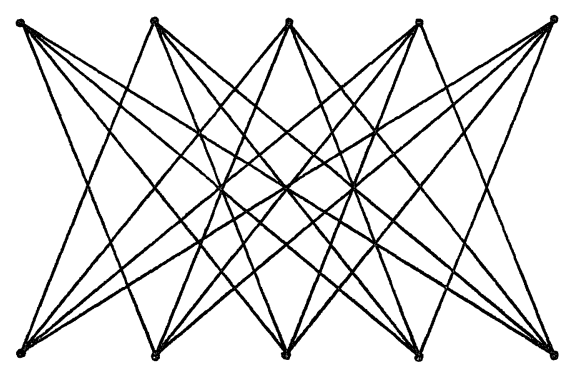

Figure 5.

Lemma 4-3. $\mathscr{M}_{2 m-1}, m=1,2,3$ are primitive tame connexes whose division spaces are $\mathscr{M}_{2 m-1}^{+}$.

Lemma 4-4. An immersion from $\mathscr{M}_{1}, \mathscr{M}_{3}$ or $\mathscr{M}_{5}$ is a $\nabla$-immersion.

These lemmata are easily verified and the proofs are omitted (see Figure 6).
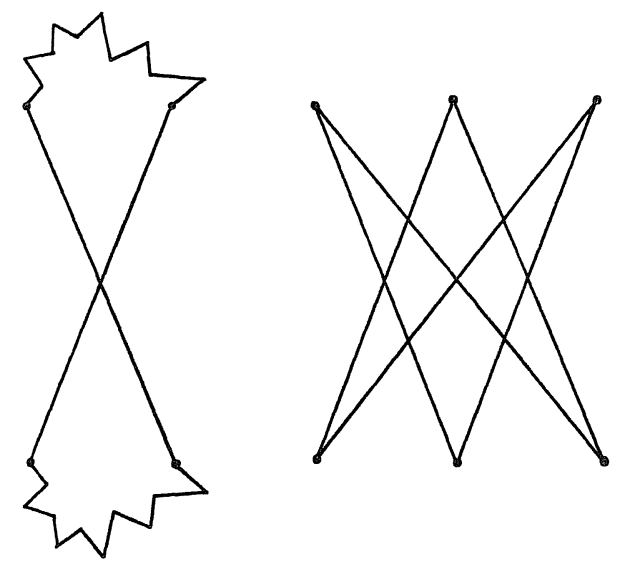

Figure 6. 
Proposition 2. For $m=1,2,3, \mathscr{M}_{2 m-1}$ is the sole tame connex without G-invariant point whose division space is $\mathscr{M}_{2 m-1}^{+}$, up to isomorphism. For $m \geqq 4$, there is no tame connex whose division space is isomorphic to $\mathscr{M}_{2 m-1}^{+}$.

Proof. We shall show the latter assertion only. Assume that there exists a tame connex $\mathscr{C}=(\tilde{X}, \varphi)$ whose division space is isomorphic to $\mathscr{M}_{2 m-1}^{+}$for an integer $m$ such that $m \geqq 4$. Let $Y \cup\left\{x_{1}, x_{2}, x_{3}\right\}$ be a subset of $X$ consisting of $m+2$ elements. Then by Lemma $2-4,(G \backslash)^{-1}\left(Y \cup\left\{x_{i}\right\}\right)$ is cyclic for each $i$. We find points $\tilde{y}_{1}, \ldots, \tilde{y}_{m-1} ; \tilde{x}_{1}, \tilde{x}_{2}$ and $\tilde{x}_{3}$ of $(G \mid)^{-1}\left(Y \cup\left\{x_{1}, x_{2}, x_{3}\right\}\right)$ satisfying

$$
\begin{array}{ll}
G \backslash\left(\tilde{y}_{j}\right) \in Y & { }^{\forall} j \\
G \backslash\left(\tilde{x}_{i}\right)=x_{i} & { }_{i} \\
\varphi\left(\tilde{y}_{j-1}, \tilde{y}_{i}\right)=1 & 2 \leqq{ }^{\forall} j \leqq m-1 \\
\varphi\left(\tilde{x}_{i}, \tilde{y}_{1}\right)=1 & { }^{{ }_{i}}
\end{array}
$$

and

$$
\varphi\left(\tilde{y}_{m-1}, \alpha\left(\tilde{x}_{i}\right)\right)=1 \quad{ }^{\forall} i .
$$

Since $m \geqq 4$, there exists a subset $Z$ of $X$ consisting of $x_{1}, x_{2}, x_{3}, G \backslash\left(\tilde{y}_{1}\right)$ and $m-4$ other elements. Thus $(G \backslash)^{-1}(Z)$ has a ramified point $\tilde{y}_{1}$ (see Figure 7). This contradicts Lemma $2-4$. Now our proposition is verified.

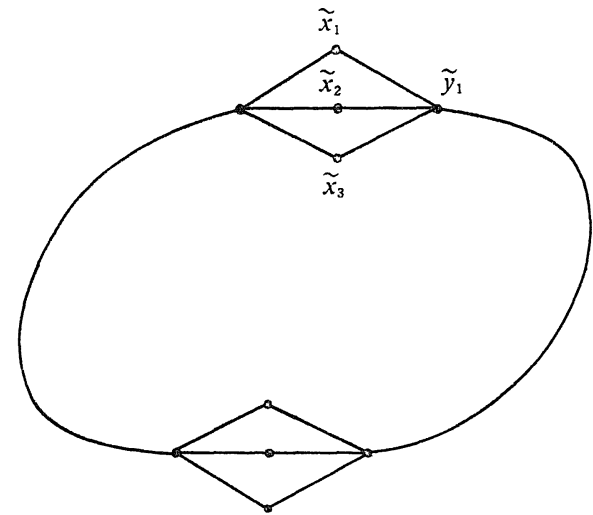

Figure 7.

Let $\mathscr{C}=(\tilde{X}, \varphi)$ be a tame connex and $\left(\tilde{x}_{0}, \tilde{x}_{1}\right)$ a pair of essential points of $\tilde{X}$ adjacent to each other. Then we obtain a tame connex $\mathscr{C}^{\prime}$ and a contraction $f$ from $\mathscr{C}$ to $\mathscr{C}^{\prime}$ such that $f\left(\tilde{x}_{0}\right)=f\left(\tilde{x}_{1}\right)$ and $f$ is injective on $\tilde{X}-G\left\{\tilde{x}_{0}, \tilde{x}_{1}\right\}$. We call $\mathscr{C}^{\prime}$ and $f$ the contracted connex and the contraction attached to $\left(\tilde{x}_{0}, \tilde{x}_{1}\right)$. We put $\tilde{F}=f^{-1}\left(\tilde{X}^{\prime} \varepsilon\right), \tilde{F}^{*}=\tilde{F}-G\left\{\tilde{x}_{0}, \tilde{x}_{1}\right\}, \tilde{Z}_{i}=\left\{\tilde{y} \in \tilde{F}^{*} \mid \varphi\left(\tilde{y}, \tilde{x}_{i}\right)=1\right\}, \tilde{Z}_{i}^{*}=\tilde{Z}_{i}-\tilde{Z}_{1-i}$ for $i=0,1$ and $\tilde{Z}=\tilde{Z}_{0} \cup \tilde{Z}_{1}$. We have 


$$
\left\{\tilde{x}_{0}, \tilde{x}_{1}\right\} \subset \tilde{F} \subset \tilde{X}^{\varepsilon}
$$

by an argument of division spaces.

Lemma 4-5. Let $\mathscr{C}=(\tilde{X}, \varphi)$ be a tame connex and $\mathscr{C}^{\prime}$ a contracted connex attached to a pair $\left(\tilde{x}_{0}, \tilde{x}_{1}\right)$. Assume that $\mathscr{C}^{\prime}$ has a $\nabla$-immersion from $\mathscr{M}_{5}$. Then $\mathscr{C}$ also has a $\nabla$-immersion from $\mathscr{M}_{5}$.

Proof. As is easily seen $|\tilde{Z}|=4$. First suppose that $\left|\tilde{Z}_{0}\right|$ or $\left|\tilde{Z}_{1}\right|$, say $\left|\tilde{Z}_{0}\right|$ is 4. Then $\left(G\left(\tilde{Z}_{0} \cup\left\{\tilde{x}_{0}\right\}\right), \varphi\right)$ is a connex isomorphic to $\mathscr{M}_{5}$ and $\tilde{x}_{1}$ must be negligible.

Next suppose $2 \leqq\left|\widetilde{Z}_{i}\right| \leqq 3$ for each $i$. Then we can divide $\tilde{Z}$ into a disjoint pair of two points subsets $\tilde{Y}_{0}$ of $\tilde{Z}_{0}$ and $\tilde{Y}_{1}$ of $\tilde{Z}_{1}$. Now we find a division $\triangleright$ on $X$ given by

$$
D \circ G \backslash(\tilde{x})=T \quad \text { iff } \quad \tilde{x} \in G\left(\tilde{Z}_{0} \cup\left\{\tilde{x}_{0}\right\}\right)
$$

Thus we have

$$
D \circ G \backslash(\tilde{x})=\perp \quad \text { if } \quad \tilde{x} \in G\left(\tilde{Z}_{1} \cup\left\{\tilde{x}_{1}\right\}\right)
$$

and therefore

$$
\mathfrak{g}[\mathfrak{d}, \mathrm{T}]=\mathfrak{g}[\mathfrak{d}, \perp] \ni G,
$$

a contradiction (see Figure 8).

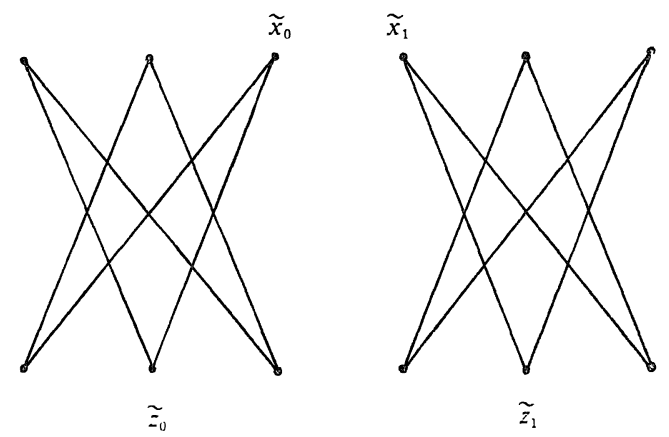

Figure 8.

Suppose finally that $\left(\left|\tilde{Z}_{0}\right|,\left|\tilde{Z}_{1}\right|\right)=(3,1)$ or $(1,3)$, say $(3,1)$. We choose an arbitrary point $\tilde{z}_{0}$ of $\tilde{Z}_{0}$ and define a division $\mathfrak{D}$ on $X$ by

$$
D \circ G \backslash(\tilde{x})=\top \quad \text { iff } \quad \tilde{x} \in G\left(\left(\tilde{Z}_{0}-\left\{\tilde{z}_{0}\right\}\right) \cup\left\{\tilde{x}_{1}\right\} \cup \tilde{A}\right)
$$

where

$$
\tilde{A}=\mathrm{Web}_{\tilde{F}}\left(\tilde{Z}_{1} \cup\left\{\tilde{x}_{0}, \tilde{x}_{1}\right\}\right)
$$


Then we easily obtain

$$
\mathfrak{g}[\mathfrak{D}, T] \not \supset G \text {. }
$$

Hence $\mathfrak{d}$ is a $\perp$-division. We have a minimal $\perp$-division $\grave{D}_{0}$ satisfying

$$
\mathfrak{D}^{-1}(\perp) \supset \mathfrak{D}_{0}^{-1}(\perp) .
$$

Since $G\left(\left(\tilde{Z}_{0}-\left\{\tilde{z}_{0}\right\}\right) \cup\{\tilde{u}\}\right)$ is connected for $\tilde{u} \in\left\{\tilde{z}_{0}, \tilde{x}_{0}, \tilde{z}_{1}\right\}$, we have

$$
\left(\mathrm{D}_{0} \circ G \backslash\right)^{-1}(\perp) \supset\left\{\tilde{z}_{0} . \tilde{x}_{0}, \tilde{z}_{1}\right\} .
$$

Then by Lemma $2-4$ there exists a chain in $\mathfrak{D}_{0}^{-1}(\perp) \subset D^{-1}(\perp)$ from $x_{0}$ to $\alpha\left(\tilde{z}_{1}\right)$, which contradicts Lemma 4-4 and the fact that $(\mathfrak{D} G \backslash)^{-1}(\perp)$ is disjoint to $\tilde{A}$ (see Figure 9). Now our lemma is verified.
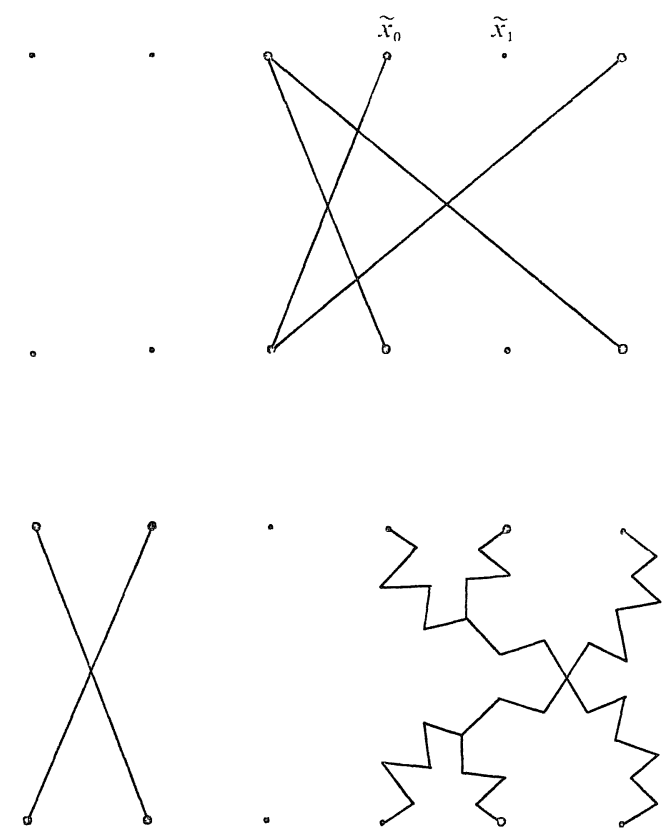

Figure 9.

A tame connex $\mathscr{C}=(\tilde{X}, \varphi)$ is said to be spherical if it is obtained by the set $\tilde{X}$ of vertices and the adjacency function $\varphi$ of a simplicial decomposition of a sphere $S^{2}$ admitting the natural action of the antipodal mapping.

Theorem $4 . \quad$ Let $\mathscr{C}$ be a tame connex. Then $\mathscr{C}$ has a $\nabla$-immersion from one of $\mathscr{M}_{,} \mathscr{M}_{1}, \mathscr{M}_{5}$ or a primitive spherical connex.

From now on in the rest of this paper, our purpose is to prove this theorem. 
Lemma 4-6. Let $\mathscr{C}=(\tilde{X}, \varphi)$ be a primitive spherical connex. Then $X$ is connected and every complete subset of $\tilde{X}$ is the set of vertices of a simplex.

Proof. The first assertion is an immediate consequence of Lemma 2-4. Let $\tilde{A}$ be a complete subset of $\tilde{X}$ consisting of three points. Then its triangle separates the sphere into two domains. Now a point is negligible if it is in the domain distinct from that containing $\alpha(\tilde{A})$ by Lemma $4-1$.

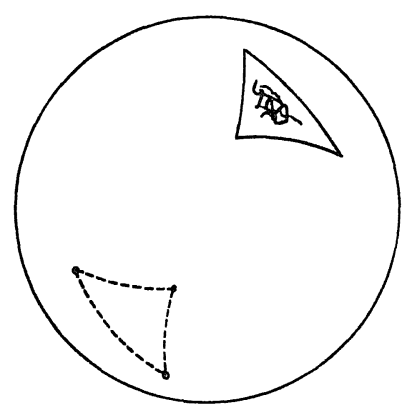

Figure 10.

Now our lemma is verified.

\section{§5. Spindle Connexes}

For any integer $n \geqq 2$, we define the spindle connex $\mathscr{S}_{2 n}=(\tilde{X}, \varphi)$ of size $2 n$ as follows (see Figure 11):

$$
\tilde{X}=\tilde{E} \cup\{\tilde{p}, \alpha(\tilde{p})\},
$$

$(\widetilde{E}, \varphi)$ is a cyclic graph consisting of $2 n$ points

where $\alpha$ acts antipodally,

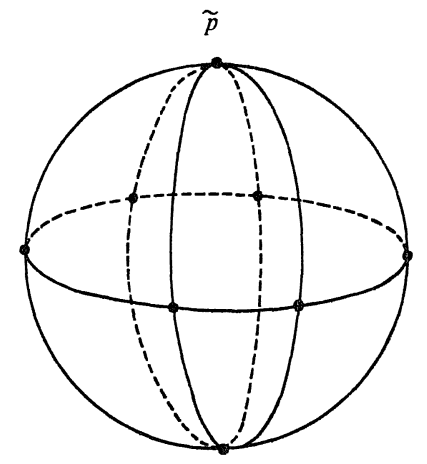

Figure 11. 
and

$$
\varphi(g(\tilde{p}), \tilde{x})=1 \quad \text { iff } \quad \tilde{x} \in \tilde{E} \text { for } g \in G .
$$

We call $\tilde{p}$ and $\alpha(\tilde{p})$ the polar points. As is easily seen $\mathscr{S}_{2 \cdot 2}$ is isomorphic to $\mathscr{M}_{3}$.

Lemma 5-1. A spindle connex is primitive and an immersion from it is a $\nabla$-immersion.

The proof is elementary and is omitted.

In the rest of this section we fix an integer $n \geqq 2$, a tame connex $\mathscr{C}=(\tilde{X}, \varphi)$ without $G$-invariant point and a pair $\left(\tilde{x}_{0}, \tilde{x}_{1}\right)$ of essential points of $\tilde{X}$ adjacent each other. Suppose that the contracted connex $\mathscr{C}^{\prime}$ has a $\nabla$-immersion $c$ from $\mathscr{S}_{2 n}$ such that 6 sends polar points to $f\left(\tilde{x}_{0}\right)=f\left(\tilde{x}_{1}\right)$ and its antipodal point where $f$ is the contraction attached to the pair $\left(\tilde{x}_{0}, \tilde{x}_{1}\right)$.

Lemma 5-2. One of the following two cases occurs.

(I) $\tilde{Z}_{i}$ is connected for $i=0,1$ and $\widetilde{Z}_{0} \cap \widetilde{Z}_{1}$ is discrete,

(II) $n=3$ and $\tilde{Z}_{i}$ is discrete for $i=0,1$.

Proof. Assume first that $\tilde{Z}_{i} \cap \alpha\left(\tilde{Z}_{i}\right)$ is empty for each $i$. Then $\tilde{Z}_{1}=\alpha\left(\tilde{Z}_{0}\right)$ and there exist a point $\tilde{z}_{0}$ of $\tilde{Z}_{0}$ and $\tilde{z}_{1}$ of $\widetilde{Z}_{1}$ adjacent each other. If $n=2$, then (I) occurs. We suppose $n \geqq 3$. Then one $\widetilde{B}$ of the two connected components of $\tilde{Z}-G\left\{\tilde{z}_{0}, \tilde{z}_{1}\right\}$ is contained in $\tilde{Z}_{0}$ and the other in $\tilde{Z}_{1}$. If it were not the case, say $\tilde{z} \in \widetilde{B} \cap \tilde{Z}_{0}$ and $\tilde{z}^{\prime} \in \alpha(B) \cap \tilde{Z}_{0}$, then there should exist a division $\mathrm{D}$ on $X$ given by

$$
\grave{D} G \backslash(\tilde{x})=\top \quad \text { iff } \quad \tilde{x} \in G\left\{\tilde{z}_{0}, \tilde{z}_{1}, \tilde{x}_{1}\right\}
$$

therefore

$$
\mathfrak{g}[\mathrm{d}, T]=\mathfrak{g}[\mathfrak{d}, \perp] \ni G
$$

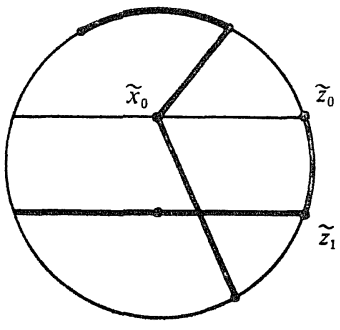

Figure 12-1.

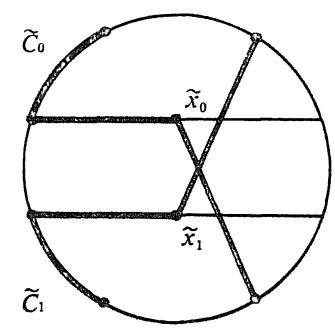

$12-2$.

a contradiction (see Figure 12-1). We may assume 


$$
\tilde{Z}_{0}=\widetilde{B} \cup\left\{\tilde{z}_{0}, \alpha\left(\tilde{z}_{1}\right)\right\}
$$

and

$$
\tilde{Z}_{1}=\alpha(\widetilde{B}) \cup\left\{\alpha\left(\tilde{z}_{0}\right), \tilde{z}_{1}\right\}
$$

If $\widetilde{Z}_{i}$ is connected for each $i$, then (I) holds. We may assume that the following $\widetilde{C}$ is connected:

$$
\tilde{C}=\widetilde{B} \cup\left\{\tilde{z}_{1}, \alpha\left(\tilde{z}_{0}\right)\right\}
$$

Suppose $n \geqq 4$. Then $\widetilde{C}$ can be divided into disjoint pair $\left(\widetilde{C}_{0}, \widetilde{C}_{1}\right)$ of connected subset $\tilde{C}_{0}$ and $\tilde{C}_{1}$ such that each of them has at least two points. Thus there exists a division $\mathfrak{D}$ on $X$ given by

$$
D \circ G \backslash(\tilde{x})=\top \quad \text { iff } \quad \tilde{x} \in G \cdot\left(\tilde{C}_{0} \cup\left\{\tilde{x}_{0}\right\}\right),
$$

hence

$$
\mathfrak{g}[\mathfrak{d}, \top]=\mathfrak{g}[\mathfrak{d}, \perp] \ni G,
$$

a contradiction. Now $n$ must be 3 and then (II) holds (see Figure 12-2).

Next assume that $\tilde{Z}_{0} \cap \alpha\left(\tilde{Z}_{0}\right)$ or $\tilde{Z}_{1} \cap \alpha\left(\tilde{Z}_{1}\right)$ is non-empty, say $\tilde{Z}_{0} \cap \alpha\left(\tilde{Z}_{0}\right)$ has a point $\tilde{z}_{0}$. We denote the two connected components of $\tilde{Z}-G\left\{\tilde{z}_{0}\right\}$ by $\tilde{B}$ and $\alpha(\widetilde{B})$. Then $\tilde{Z}_{1}$ is contained in $\widetilde{B} \cup G\left\{\tilde{z}_{0}\right\}$ or in $\alpha(\widetilde{B}) \cup G\left\{\tilde{z}_{0}\right\}$, because of the division $\mathfrak{D}$ over $X$ defined as follows (see Figure 13-1).

$$
\triangleright \circ G \backslash(\tilde{x})=\top \quad \text { iff } \quad \tilde{x} \in G\left\{\tilde{z}_{0}, \tilde{x}_{0}\right\} .
$$

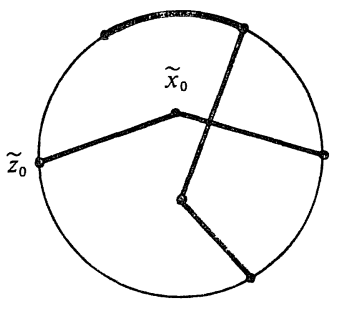

Figure 13-1.

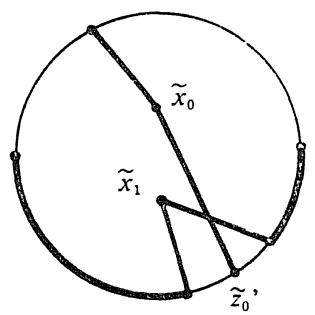

13-2.

We may assume that $\tilde{Z}_{1}$ is contained in $\widetilde{B} \cup G\left\{\tilde{z}_{0}\right\}$. If there exists $\tilde{z}_{0}^{\prime} \in \tilde{Z}_{0}$ such that each of the two connected components of $\tilde{Z}-G\left\{\tilde{z}_{0}^{\prime}\right\}$ has non-empty intersection with $\tilde{Z}_{1}$, then

$$
\mathfrak{g}[\mathrm{d}, \mathrm{T}]=\mathrm{g}[\mathrm{D}, \perp] \ni G
$$

for the division $\mathfrak{D}$ on $X$ given by 


$$
\mathfrak{D} \circ G \backslash(\tilde{x})=\top \quad \text { iff } \quad \tilde{x} \in G\left\{\tilde{z}_{0}^{\prime}, \tilde{x}_{0}\right\} .
$$

Now we obtain (I) and our lemma is verified (see Figure 13-2).

Lemma 5-3. In case (II), $\mathscr{C}$ has a $\nabla$-immersion from $\mathscr{H}_{5}$.

This lemma is an immediate consequence of Lemma 4-3 (see Figure 14).

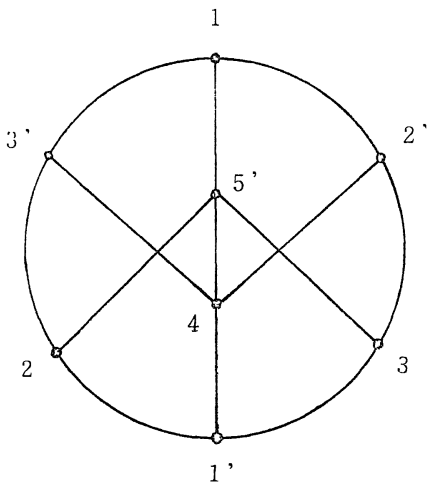

Figure 14.

Lemma 5-4. Assume that (I) occurs. Let $\tilde{C}$ be a connected subset of $\tilde{X}-\tilde{F}$. Assume that $\operatorname{Supp}_{\tilde{F}}(\tilde{C})$ contains $\tilde{x}_{1}\left(\operatorname{resp} . \tilde{x}_{0}\right)$ and a point $\tilde{z}_{0} \in \tilde{Z}_{0}$ (resp. $\tilde{z}_{1} \in \tilde{Z}_{1}$ ). Then $\tilde{z}_{0}$ (resp. $\tilde{z}_{1}$ ) is a terminal point of $\tilde{Z}_{0}$ (resp. $\tilde{Z}_{1}$ ).

Proof. Suppose that the assertion does not hold, say $\tilde{x}_{1}$ and a non-terminal point $\tilde{z}_{0}$ of $\tilde{Z}_{0}$ is contained in $\operatorname{Supp}_{\tilde{F}}(\tilde{C})$. If $\alpha\left(\tilde{z}_{0}\right) \in \tilde{Z}_{1}$, then we have

$$
\mathfrak{g}[\mathfrak{D}, T]=\mathfrak{g}[\mathfrak{D}, \perp] \ni G
$$

for the following division $\mathrm{D}$ on $X$ (see Figure 15-1):

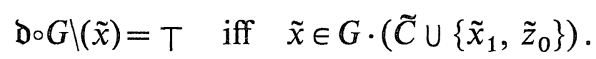

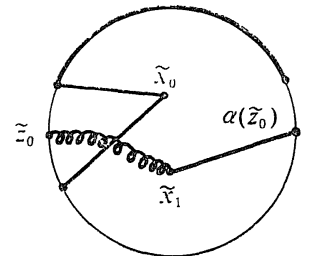

Figure 15-1.

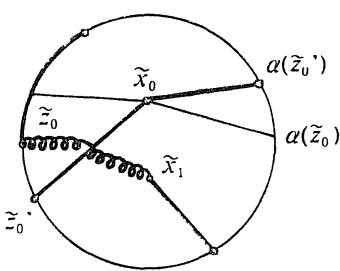

$15-2$.

If $\alpha\left(\tilde{z}_{0}\right) \notin \tilde{Z}_{1}$, then $\alpha\left(\tilde{z}_{0}\right) \in \tilde{Z}_{0}$ and we choose a point $\tilde{z}_{0}^{\prime} \in \tilde{Z}_{0}$ adjacent to $\tilde{z}_{0}$ so that it is in the same connected component of $\tilde{Z}-\left\{\tilde{z}_{0}, \alpha\left(\tilde{z}_{0}\right)\right\}$ with $\tilde{Z}_{1}$. In such a 
case, we define the following division $\mathfrak{D}$ on $X$ :

$$
\grave{D} \cdot G \backslash(\tilde{x})=\top \quad \text { iff } \quad \tilde{x} \in G\left\{\tilde{x}_{0}, \tilde{z}_{0}^{\prime}\right\}
$$

and obtain

$$
\mathrm{g}[\mathrm{d}, \mathrm{T}]=\mathrm{g}[\mathrm{d}, \perp] \in G,
$$

because $\tilde{Z}_{1}^{*}$ is non-empty. Now our lemma is verified (see Figure 15-2).

Lemma 5-5. Assume that (I) occurs. Let $\tilde{C}$ be a connected subset of $\tilde{X}-\tilde{F}$. Then $\operatorname{Supp}_{\tilde{F}}(\widetilde{C})$ is complete or

$$
\left.\begin{array}{l}
\operatorname{Supp}_{\tilde{F}}(\tilde{C}) \subset\left\{\tilde{x}_{0}, \tilde{x}_{1}, \tilde{z}_{0}, \tilde{z}_{1}\right\} \\
\text { for a point } \tilde{z}_{0} \in \tilde{Z}_{0}^{*} \text { and a point } \\
\tilde{z}_{1} \in \tilde{Z}_{1}^{*} \text { adjacent to it }
\end{array}\right\} \cdots \cdots(*)
$$

Proof. We suppose that our assertion does not hold. By Lemma 5-1, $f\left(\operatorname{Supp}_{\tilde{F}}(\widetilde{C})\right)$ is complete. For conveniences sake, we may assume

$$
\left\{\tilde{x}_{1}, \tilde{z}_{0}\right\} \subset \operatorname{Supp}_{\tilde{F}}(\tilde{C}) \subset\left\{\tilde{x}_{0}, \tilde{x}_{1}, \tilde{z}_{0}, \tilde{z}_{0}^{\prime}\right\}
$$

where $\tilde{z}_{0} \in \tilde{Z}_{0}^{*}$ and $\tilde{z}_{0}^{\prime}$ is a point of $\tilde{Z}_{0}$ adjacent to $\tilde{z}_{0}$. Then by the above lemma, $\tilde{z}_{0}$ is adjacent to a point of $\tilde{Z}_{1}^{*}$. If

$$
\operatorname{Supp}_{\tilde{F}}(\tilde{C}) \subset\left\{\tilde{x}_{0}, \tilde{x}_{1}, \tilde{z}_{0}\right\},
$$

then $(*)$ occurs. We assume

$$
\left\{\tilde{x}_{1}, \tilde{z}_{0}, \tilde{z}_{0}^{\prime}\right\} \subset \operatorname{Supp}_{\tilde{F}}(\tilde{C}) .
$$

Then $\tilde{Z}_{0}=\left\{\tilde{z}_{0}, \tilde{z}_{0}^{\prime}\right\}$ by the above lemma. Therefore we have

$$
\alpha\left(\tilde{z}_{0}\right) \subset \tilde{Z}_{1}
$$

Case (1). Assume that there exists a connected subset $\widetilde{C}^{\prime}$ of $\tilde{X}-\tilde{F}$ such that

$$
\left\{\tilde{x}_{0}, \tilde{z}_{1}\right\} \subset \operatorname{Supp}_{\tilde{F}}\left(\tilde{C}^{\prime}\right)
$$

First we show that $\tilde{C}^{\prime}$ is disjoint to $\tilde{C}$. We may assume that $\tilde{C}^{\prime}$ is chosen maximally. If $\tilde{C}^{\prime}$ intersects $\tilde{C}$, then $\tilde{C}^{\prime} \supset \widetilde{C}$ and we have $\tilde{C}^{\prime} \supset\left\{\tilde{z}_{0}^{\prime}, \tilde{z}_{1}\right\}$ which contradicts the fact that $f\left(\operatorname{Supp}_{\tilde{F}}\left(\tilde{C}^{\prime}\right)\right)$ is complete. Now $\tilde{C}^{\prime}$ must be disjoint to $\tilde{C}$. Then we have

$$
\mathfrak{g}[\mathrm{D}, \mathrm{T}]=\mathfrak{g}[\mathrm{D}, \perp] \ni G
$$

for a division $\mathfrak{b}$ on $X$ defined as follows (see Figure 16-1):

$$
\triangleright \circ G \backslash(\tilde{x})=\top \quad \text { iff } \quad \tilde{x} \in G\left(\tilde{C} \cup\left\{\tilde{x}_{1}, \tilde{z}_{0}\right\}\right)
$$




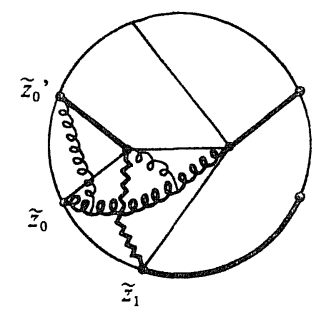

Figure 16-1.

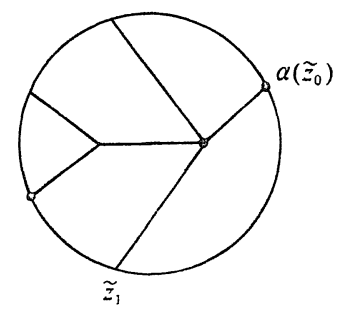

$16-2$.

Case (2). Assume that there is not subset of $\tilde{X}-\widetilde{F}$ whose support contains $\left\{\tilde{x}_{0}, \tilde{z}_{1}\right\}$. We define a division $\mathfrak{D}$ on $X$ by

$$
\mathfrak{D} \circ G \backslash(\tilde{x})=\top \quad \text { iff } \quad \tilde{x} \in G\left\{\tilde{x}_{1}, \tilde{z}_{0}\right\} .
$$

Then we easily have

$$
g[D, T] \not \nexists G .
$$

Hence $\mathfrak{D}$ is a $\perp$-division. We have a minimal $\perp$-division $\mathfrak{D}_{0}$ satisfying

$$
D_{0}^{-1}(\perp) \subset D^{-1}(\perp) \text {. }
$$

Since $G\left\{\tilde{x}_{1}, \tilde{z}_{0}, \tilde{x}_{0}\right\}$ and $G\left(\left\{\tilde{x}_{1}\right\} \cup \tilde{Z}\right)$ are connected, $\left(\mathfrak{D}_{0} \circ G \backslash\right)^{-1}(\perp)$ contains $\tilde{x}_{0}$ and intersects $\tilde{Z}$. Then by Lemma $2-4$ there exists a chain in $\mathfrak{D}_{0}^{-1}(\perp) \subset \mathfrak{D}^{-1}(\perp)$ from $\tilde{x}_{0}$ to a point of the connected component of $\tilde{Z}-G\left\{\tilde{z}_{1}\right\}$ not containing $\tilde{z}_{0}^{\prime}$, which contradicts the above lemma and the fact that there is no point of $\tilde{X}-\tilde{F}$ whose support contains $\tilde{x}_{0}$ and $\tilde{z}_{1}$. Now our lemma is verified (see Figure 16-2).

\section{§6. Spherical Connexes}

In this section we study a contraction where the contracted connex has a $\nabla$-immersion from a primitive spherical connex besides the ones treated in the last section. We fix a tame connex $\mathscr{C}=(\tilde{X}, \varphi)$ without $G$-invariant point. We assume that the contracted connex has a $\nabla$-immersion from a primitive spherical connex for each pair of essential points of $\tilde{X}$ adjacent each other. We fix also a pair $\left(\tilde{x}_{0}, \tilde{x}_{1}\right)$ of essential points of $\tilde{X}$ adjacent to each other. For this pair we assume $\alpha(\tilde{Z}) \neq \tilde{Z}$.

Let $\mathscr{C}_{0}=\left(\tilde{X}_{0}, \varphi_{0}\right)$ be a connex having a $\nabla$-immersion from a primitive spherical connex and $\widetilde{S}$ a subset of $\tilde{X}_{0}^{\varepsilon}$. Then we denote by $[\tilde{S}]$ the subset of the unit sphere $S^{2}$ covered by the simplexes whose vertices are in $\tilde{S}$.

Lemma 6-1. Assume $\alpha(\tilde{Z}) \cap \tilde{Z}=\varnothing$. Then $[f(\tilde{F})]$ is homeomorphic to 
the product $\left[\begin{array}{ll}0 & 1\end{array}\right] \times S^{1}$ of the closed interval and the unit circle, where its boundary is $[f(\tilde{Z})] \cup[\alpha f(\tilde{Z})]$.

This lemma is verified by elementary argument of topology and its proof is omitted (see Figure 17).

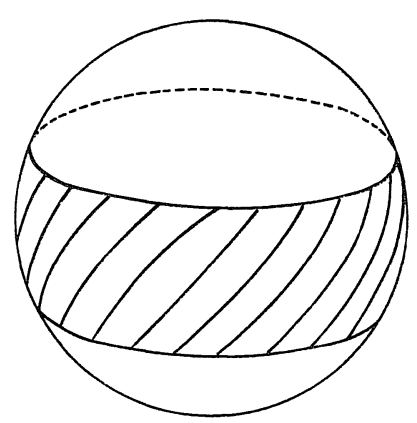

Figure 17.

Lemma 6-2. Assume $\alpha(\tilde{Z}) \cap \widetilde{Z} \neq \varnothing$. Then the number of connected components of $\tilde{Z}-\alpha(\tilde{Z})$ coincides with that of $\alpha(\tilde{Z}) \cap \tilde{Z}$, which is an even number.

Proof. The upper half of this lemma is obvious since $\tilde{Z}$ is cyclic. The number of the connected components of $\alpha(\tilde{Z}) \cap \tilde{Z}$ is even, because $\alpha(\tilde{Z}) \cap \tilde{Z}$ is $G$-invariant and has no $G$-invariant connected component.

Lemma 6-3. Assume that $\alpha(\tilde{Z}) \cap \tilde{Z}$ has 21 connected components for a positive integer $l$. Then $\tilde{F}^{*}-\alpha(\tilde{Z}) \cap \tilde{Z}$ has $2 l$ connected components. Let $\tilde{S}$ be a connected component of $\tilde{F}^{*}-\alpha(\tilde{Z}) \cap \tilde{Z}$. Then there are two points $\tilde{u}$ and $\tilde{v}$ of $\alpha(\tilde{Z}) \cap \tilde{Z}$ adjacent to a point of $\tilde{S}$ in each, where the connected component of $\alpha(\tilde{Z}) \cap \tilde{Z}$ containing $\tilde{u}$ is disjoint to that containing $\tilde{v}$. $[f(\tilde{S} \cup\{u, v\})]$ is homeomorphic to the closed square $[01]^{2}$, where its boundary is $\left[f\left(\tilde{S}_{1} \cup\right.\right.$ $\{\tilde{u}, \tilde{v}\})] \cup\left[f\left(\tilde{S}_{2} \cup\{u, v\}\right)\right]$ for a connected component $\tilde{S}_{1}$ of $\tilde{Z}-\alpha(\tilde{Z})$ and one $\tilde{S}_{2}$ of $\alpha(\tilde{Z})-\tilde{Z}$.

Proof. We observe the embedding of $\tilde{X}^{\prime \varepsilon}$ into the unit sphere $S^{2}$. The complement of $\left[f\left((\alpha(\tilde{Z}) \cap \tilde{Z}) \cup G\left\{\tilde{x}_{0}\right\}\right)\right]$ in $S^{2}$ is a union of $2 l$ open squares (see Figure 18-1).

Thus our lemma is verified by an elementary argument of topology (see Figure 18-2). 


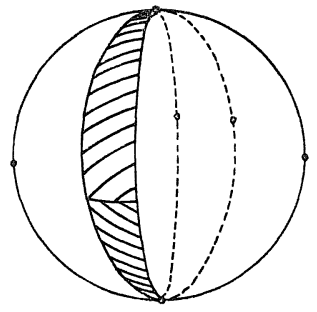

Figure 18-1.

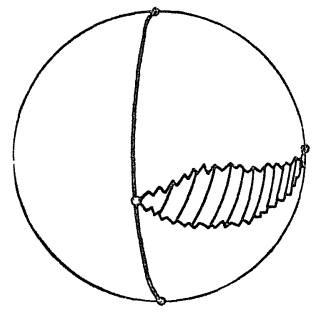

$18-2$.

Lemma 6-4. Let $\tilde{u}$ be a point of $\tilde{Z}-\alpha(\tilde{Z}), \tilde{v}$ a point of $\tilde{Z}$ non-adjacent to $\tilde{u}$, and $\tilde{w}$ a point of $\tilde{F}^{*}-\tilde{Z}$ adjacent to both $\tilde{u}$ and $\tilde{v}$. Then $\tilde{F}^{*}-\{\tilde{u}, \tilde{v}, \tilde{w}\}$ has two connected components $\widetilde{Q}$ and $\widetilde{R}$, where $[f(\tilde{Q} \cup\{\tilde{u}, \tilde{v}, \tilde{w}\})]$ or $[f(\tilde{R} \cup\{\tilde{u}, \tilde{v}$, $\tilde{w}\})]$, say the former, is homeomorphic to the closed square $\left[\begin{array}{ll}0 & 1\end{array}\right]^{2}$ and the other is not.

This lemma follows immediately Lemma 6-1 and Lemma 6-3. We denote the above component by $\widetilde{Q}(\tilde{u}, \tilde{v} ; \tilde{w})$ (see Figure 19 ).

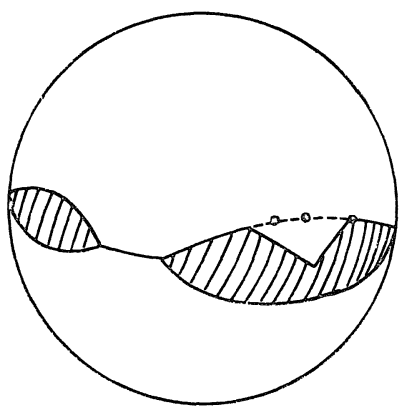

Figure 19.

Let $\mathfrak{p}=\left(i, \tilde{z}_{i}\right)$ be a pair of $i \in\{0,1\}$ and $\tilde{z}_{i} \in \tilde{Z}_{i}-\alpha(\tilde{Z})$. Then we call it a place. We denote by $\mathscr{C}_{p}=\left(\tilde{X}_{p}, \varphi_{p}\right)$ the contracted connex of $\mathscr{C}$ and by $f_{p}$ the contraction attached to the pair $\left(\tilde{z}_{i}, \tilde{x}_{i}\right)$. We define $\tilde{F}_{\mathfrak{p}}=f_{p}^{-1}\left(\tilde{X}_{\mathfrak{p}}^{\varepsilon}\right)$ and $\tilde{F}_{p}^{*}$ $=\widetilde{F}_{p}-G\left\{\tilde{z}_{i}, \tilde{x}_{i}\right\}$. The place $\mathfrak{p}$ is said to be of type $\mathbb{A}$ if

$$
\tilde{x}_{1-i} \in \tilde{F}_{\mathfrak{p}},
$$

of type $B$ if

$$
\tilde{x}_{1-i} \notin \widetilde{F}_{\mathfrak{p}} \wedge \widetilde{Z}_{1-i} \not \subset \widetilde{F}_{\mathfrak{p}}
$$

and of type $\mathbb{C}$ if

$$
\tilde{x}_{1-i} \notin \widetilde{F}_{\mathfrak{p}} \wedge \widetilde{Z}_{1-i} \subset \widetilde{F}_{p}
$$


In the rest of this section we fix the place $p$ also.

Lemma 6-5. Let $\tilde{y}$ be a point of $\tilde{F}^{*}-\tilde{F}_{\mathfrak{p}}$. Then there exists an element $g \in G$ such that

$$
\operatorname{Supp}_{\tilde{F}_{p}}(\{\tilde{y}\})=g\left\{\tilde{x}_{\boldsymbol{i}}, \tilde{z}_{\boldsymbol{i}}, \tilde{z}, \tilde{w}\right\}
$$

where $\tilde{z}$ is a point of $\tilde{Z}_{i}$ non-adjacent to $\tilde{z}_{i}$ and $\tilde{w}$ is a point of $\tilde{F}^{*}-\tilde{Z}$ adjacent to both $\tilde{z}_{i}$ and $\tilde{z}$. Furthermore

$$
\tilde{F}^{*} \cap \mathrm{Web}_{\widetilde{F}_{\mathfrak{p}}}\left(\operatorname{Supp}_{\tilde{F}_{\mathfrak{p}}}(\{\tilde{y}\})\right)=g \widetilde{Q}\left(\tilde{z}_{i}, \tilde{z}, \tilde{w}\right) .
$$

Proof. We put $\tilde{S}=\operatorname{Supp}_{\tilde{F}_{\mathfrak{p}}}(\{\tilde{y}\})$, which is not complete by Lemma 4-1. Then we define $g$ so that $f_{p}(\tilde{S})$ contains $g f_{p}\left(\tilde{x}_{i}\right)$. For instance we assume $g=1$. If there is a chain $\tilde{w}_{1}^{\prime}, \ldots, \tilde{w}_{l-1}^{\prime}$ in $f\left(\tilde{F}^{*}-\tilde{S}\right)$ from $f(\tilde{y})$ to $\alpha f\left(\tilde{x}_{i}\right)$, then we choose $\tilde{w}_{j}$ for $1 \leqq j \leqq l-1$ so that $f\left(\tilde{w}_{j}\right)=\tilde{w}_{j}^{\prime}$, where $\tilde{w}_{1}, \ldots, \tilde{w}_{l-1}$ or $\tilde{w}_{1}, \ldots, \tilde{w}_{l-1}, \tilde{x}_{1-i}$ form a chain in $\tilde{F}-\tilde{S}$ from $\tilde{y}$ to a point $\alpha\left(\tilde{x}_{i}\right)$ of $\tilde{F}_{p}$ and this contradicts the fact that $\tilde{S}$ is the support of $\{\tilde{y}\}$.

We assume that there exists a point $\tilde{w}$ of $\tilde{S}-\bar{W}_{\tilde{F}}\left(\tilde{Z} \cup\left\{\tilde{x}_{0}, \tilde{x}_{1}\right\}\right)$, because we can find a chain mentioned above if there is not such a point. Then $\tilde{w}$ is not adjacent to $\tilde{x}_{i}$, since $\tilde{w} \in \tilde{F}^{*}-\tilde{Z}$. We assume that there exists a point $\tilde{z}$ of $\tilde{S}-\left\{\tilde{x}_{i}, \tilde{z}_{i}, \tilde{w}\right\}$, if it were not the case we can find a chain as above (see Figure 20-1). If $\tilde{z}$ is adjacent to $\tilde{z}_{i}$, then $f\left(\left\{\tilde{z}_{i}, \tilde{z}, \tilde{w}\right\}\right)$ is the set of vertices of a 2-simplex by Lemma $4-6$ and we can find a chain as above. Then $\tilde{z}$ is not adjacent to $\tilde{z}_{i}$, hence $\tilde{z} \in \tilde{Z}_{i}$. Now we have

$$
\tilde{S} \subset\left\{\tilde{x}_{i}, \tilde{z}_{i}, \tilde{z}, \tilde{w}\right\}
$$

We obtain

$$
\tilde{y} \in \tilde{Q}\left(\tilde{z}_{i}, \tilde{z} ; \tilde{w}\right),
$$

if it were not the case we can find a chain as above (see Figure 20-2).

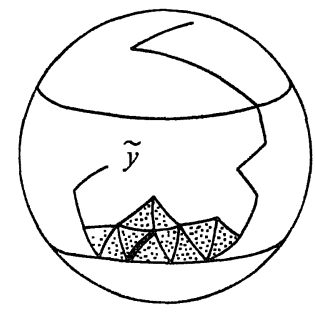

Figure 20-1.

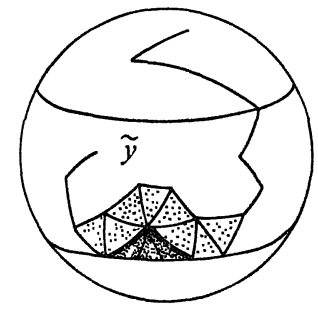

20-2.

Since $\widetilde{Q}\left(\tilde{z}_{i}, \tilde{z} ; \tilde{w}\right)$ and $\widetilde{Q}\left(\tilde{z}_{i}, \tilde{z} ; \tilde{w}\right) \cup\left\{\tilde{x}_{0}, \tilde{x}_{1}\right\}$ are connected we have 


$$
\tilde{S}=\left\{\tilde{x}_{i}, \tilde{z}_{i}, \tilde{z}, \tilde{w}\right\}
$$

and

$$
\tilde{F}^{*} \cap \operatorname{Web}_{\tilde{F}_{\mathfrak{p}}}\left(\operatorname{Supp}_{\tilde{F}_{\mathfrak{p}}}(\{\tilde{y}\})\right)=\widetilde{Q}\left(\tilde{z}_{i}, \tilde{z} ; \tilde{w}\right) \text {. }
$$

Lemma 6-6. Let $\tilde{z}$ be a point of $\tilde{Z}_{i}$ non-adjacent to $\tilde{z}_{i}$ and $\tilde{w}$ a point of $\tilde{F}^{*}-\tilde{Z}$ adjacent to both $\tilde{z}_{i}$ and $\tilde{z}$. Then we have

$$
\tilde{Q}\left(\tilde{z}_{i}, \tilde{z} ; \tilde{w}\right) \cap \tilde{F}_{p}=\varnothing .
$$

Proof. We may assume that $\tilde{z}$ and $\tilde{w}$ are taken so that $\tilde{Q}\left(\tilde{z}_{i}, \tilde{z} ; \tilde{w}\right)$ is maximal. Then $\{\tilde{z}, \tilde{w}\} \subset \tilde{F}_{\mathfrak{p}}$ by the above lemma. There are exactly two points $\tilde{u}_{1}$ and $\tilde{u}_{2}$ of $\tilde{F}^{*}$ adjacent to both $\tilde{z}_{i}$ and $\tilde{w}$ since $f\left(\tilde{z}_{i}\right)$ and $f(\tilde{w})$ are essential points of a primitive spherical connex. We define a point $\tilde{v}_{j}$ for $j=1,2$ by

$$
\left\{\tilde{v}_{j}\right\}=\left\{\begin{array}{l}
\left\{\tilde{u}_{j}\right\} \quad \text { if } \quad \tilde{u}_{j} \in \tilde{F}_{\mathfrak{p}} \\
\operatorname{Supp}_{\tilde{F}_{\mathfrak{p}}}\left(\left\{\tilde{u}_{j}\right\}\right)-\left\{\tilde{z}_{i}, \tilde{x}_{i}, \tilde{w}\right\} \text { if } \tilde{u}_{j} \notin \tilde{F}_{\mathfrak{p}} .
\end{array}\right.
$$

Since $\mathscr{C}_{\mathfrak{p}}$ has a $\nabla$-immersion from a primitive spherical connex, there exists $j$ such that $\tilde{z}=\tilde{v}_{j}$, where $\tilde{u}_{j} \notin \tilde{F}_{p}$. We put $\tilde{y}=\tilde{u}_{j}$ to apply the above lemma. Now our lemma is verified.

Lemma 6-7. There exists a homeomorphism from $S^{2}$ to $S^{2}$ which sends $\left[f\left(\tilde{F}^{*} \cap \tilde{F}_{p}\right)\right]$ to $\left[f_{p}\left(\tilde{F}^{*} \cap \tilde{F}_{p}\right)\right]$.

Proof. We see that $\tilde{F}^{*} \cap \widetilde{F}_{p}$ is connected and that its complement in $\tilde{F}$ has at most two connected components. Then we obtain our conclusion taking care of the orientation of every face (see Figure 21).

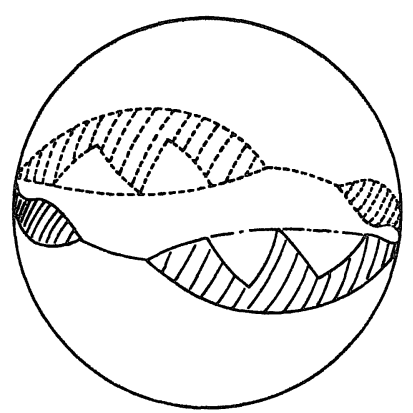

Figure 21.

Lemma 6-8. Assume that $\mathfrak{p}$ is of type C. Then $\tilde{Z}_{1-i}^{*}$ consists of a point $\tilde{z}_{1-i}$ adjacent to $\tilde{z}_{i}$ and any other point of $\tilde{Z}_{1-i}$ is adjacent to $\tilde{z}_{1-i}$ (see Figure 22). 


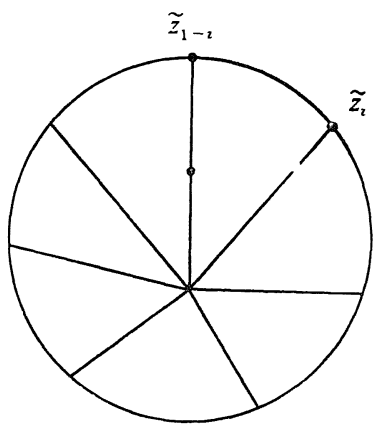

Figure 22.

Proof. We see that $\operatorname{Supp}_{\tilde{F}_{\mathfrak{p}}}\left(\left\{\tilde{x}_{1-i}\right\}\right)$ contains $\tilde{Z}_{1-i} \cup\left\{\tilde{x}_{i}\right\}$. Since $\tilde{x}_{1-i}$ is an essential point, $\tilde{Z}_{1-i}^{*}$ is non-empty. On the other hand, a point of it is adjacent to $\tilde{z}_{i}$ and points of $\tilde{Z}_{1-i}-\left\{\tilde{z}_{i}\right\}$ are adjacent to each other, because $f_{\mathfrak{p}}\left(\operatorname{Supp}_{\tilde{F}_{\mathfrak{p}}}\left(\left\{\tilde{x}_{1-i}\right\}\right)\right)$ is complete. Now our lemma is verified.

Lemma 6-9. Assume that $\mathfrak{p}$ is of type B. Then $\widetilde{Z}_{1-i}^{*}$ is disjoint to $\alpha(\tilde{Z})$ and $\left(1-i, \tilde{z}_{1-i}\right)$ is of type A for any point $\tilde{z}_{1-i}$ of $\tilde{Z}_{1-i}^{*}$.

This lemma is an immediate consequence of Lemma 6-5 (see Figure 23).

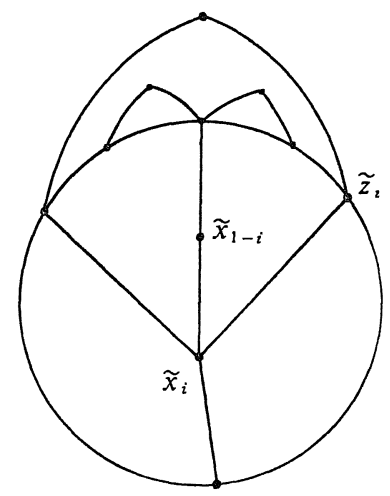

Figure 23.

Lemma 6-10. $\tilde{Z}_{0}$ and $\tilde{Z}_{1}$ are connected and $\tilde{Z}_{0} \cap \widetilde{Z}_{1}$ is discrete.

Proof. If $\mathfrak{p}$ is of type $\mathrm{C}$, then our conclusion holds by Lemma 6-8. We may assume that $\mathfrak{p}$ is of type $A$ by the above lemma. Hence $\tilde{Z}_{1-i} \subset \tilde{F}_{\mathfrak{p}}^{*}$ by Lemma 6-5. Now our assertion holds by Lemma 6-7 and the fact that $\mathscr{C}_{p}$ has a $\nabla$-immersion from a primitive spherical connex (see Figure 24). 


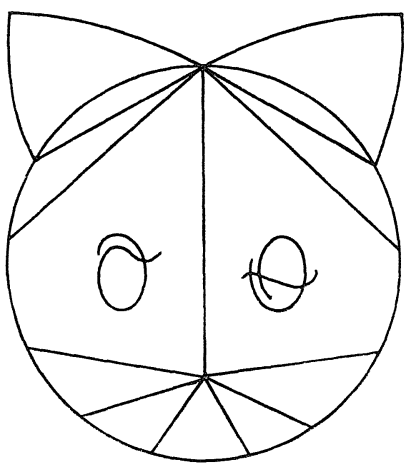

Figure 24.

Lemma 6-11. Let $\widetilde{C}$ be a connected subset of $\operatorname{Web}_{\tilde{F}}\left(\tilde{Z} \cup\left\{\tilde{x}_{0}, \tilde{x}_{1}\right\}\right)$. We put $\tilde{S}=\operatorname{Supp}_{\tilde{F}}(\widetilde{C})$. Assume that $f_{p}(\tilde{S})$ is not a complete set of at most three essential points. Then one of the following three cases occurs:

i) $\tilde{S} \subset \bar{W}_{\tilde{F}_{\tilde{F}} \widetilde{F}_{\mathfrak{p}}}\left(\operatorname{Supp}_{\tilde{F}_{\tilde{F}} \cap \widetilde{F}_{\mathfrak{p}}}(\tilde{A})\right)$ where $\tilde{A}$ is a connected subset of $\tilde{Z}-\widetilde{F}_{p}$,

ii) $\mathfrak{p}$ is of type A and $\tilde{S} \subset\left\{\tilde{z}_{i}, \tilde{x}_{0}, \tilde{x}_{1}, \tilde{u}_{0}, \tilde{u}_{1}\right\}$ where $\left(\tilde{u}_{0}, \tilde{u}_{1}\right) \in \tilde{Z}_{0}^{*} \times \tilde{Z}_{1}^{*}$ and $\tilde{u}_{1}$ is adjacent to $\tilde{u}_{0}$,

iii) $\mathfrak{p}$ is of type $\mathrm{C}$ and $\tilde{S} \subset\left\{\tilde{z}_{i}, \tilde{x}_{0}, \tilde{x}_{1}, \tilde{z}_{1-i}, \tilde{z}\right\}$ where $\tilde{z}_{1-i} \in \tilde{Z}_{1-i}^{*}$ and $\tilde{z}$ is the terminal point of $\tilde{Z}_{i}$ besides $\tilde{z}_{i}$.

Proof. We observe $\mathscr{C}_{p}$ and obtain our assertion by Lemma 4-2, Lemma 6-7 and the above lemma, according to the type of $\mathfrak{p}$ (see Figure 25).

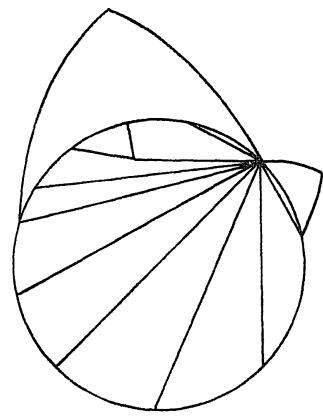

Figure 25-1.

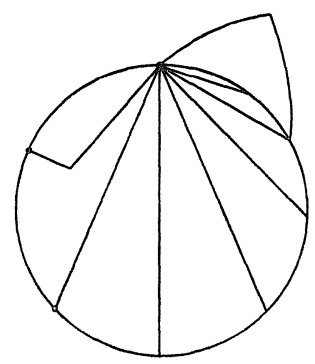

25-2.

\section{§7. The Proof of Theorem 4}

In the rest of this paper, we shall prove our Theorem 4 by induction on the dictionary order of the number of vertices and edges. Let $\mathscr{C}=(\tilde{X}, \varphi)$ be a tame 
connex. We assume that the induction hypothesis holds for any tame connex with smaller order and that $\mathscr{C}$ has no $\nabla$-immersion from $\mathscr{M}, \mathscr{M}_{1}$ or $\mathscr{M}_{5}$. We choose a minimal $T$-division $D_{0}$ on $X$ so that $\left|\mathfrak{D}_{0}^{-1}(T)\right|$ is maximal. By Lemma 4-4 and Proposition $2,\left|\mathfrak{D}_{0}^{-1}(T)\right| \geqq 2$. If $\left|\mathfrak{D}_{0}^{-1}(T)\right|=2$, then $\mathscr{C}$ has a $\nabla$-immersion from $\mathscr{M}_{3}$ by Lemma 4-4 and Proposition 2. In the rest of this paper, we assume $\left|\mathfrak{D}_{0}^{-1}(T)\right| \geqq 3$. We fix a pair $\left(\tilde{x}_{0}, \tilde{x}_{1}\right)$ of points of $(\mathfrak{D} \circ G)^{-1}(T)$ adjacent to each other. We consider the contraction $f$ attached to this pair and adopt the notions in Section 4 and Section 6.

Lemma 7-1. Assume $\tilde{Z}=\alpha(\tilde{Z})$. Then $\mathscr{C}$ has a $\nabla$-immersion from a spherical connex.

Proof. By Lemma 5-5, we study $\bar{W}_{\tilde{F}}\left(\left\{\tilde{z}_{0}, \tilde{z}_{1}, \tilde{x}_{0}, \tilde{x}_{1}\right\}\right)$ for $\left(\tilde{z}_{0}, \tilde{z}_{1}\right) \in \tilde{Z}_{0}^{*}$ $\times \tilde{Z}_{1}^{*}$ where $\tilde{z}_{1}$ is adjacent to $\tilde{z}_{0}$. First suppose $\left|\tilde{Z}_{0}^{*}\right|=1$ or $\left|\tilde{Z}_{1}^{*}\right|=1$, say $\tilde{Z}_{0}^{*}=\left\{\tilde{z}_{0}\right\}$. Then contracting the two connected components of $\tilde{Z}-\left\{\tilde{z}_{0}, \alpha\left(\tilde{z}_{0}\right)\right\}$ in each and $\tilde{x}_{1}$ to $\alpha\left(\tilde{z}_{0}\right)$, we obtain a connex, which has a $\nabla$-immersion from a primitive spherical connex since the images of $\tilde{z}_{0}, \tilde{x}_{0}$ and the terminal points of $\tilde{Z}_{1}$ remain essential (see Figure 26-1).

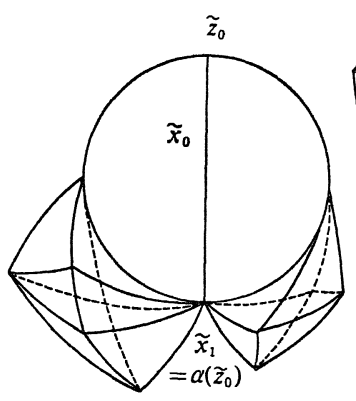

Figure 26-1.

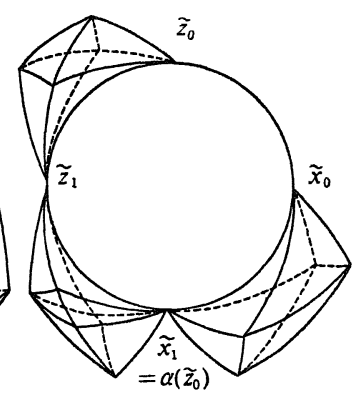

26-2.

In this case our assertion holds.

Next suppose $\left|\tilde{Z}_{i}^{*}\right| \geqq 2$ for $i=0,1$. Let $\left(\tilde{z}_{0}, \tilde{z}_{1}\right) \in \tilde{Z}_{0}^{*} \times \tilde{Z}_{1}^{*}$ and assume that $\tilde{z}_{1}$ is adjacent to $\tilde{z}_{0}$. Then $\alpha\left(\tilde{z}_{0}\right) \in \tilde{Z}_{1}$ or $\alpha\left(\tilde{z}_{1}\right) \in \tilde{Z}_{0}$, say the former. Contracting $\tilde{x}_{1}$ to $\alpha\left(\tilde{z}_{0}\right)$, the connected component of $\tilde{Z}-G\left\{\tilde{z}_{0}\right\}$ intersecting $\tilde{Z}_{0}$ to $\tilde{x}_{0}$ and the other component to $\tilde{z}_{1}$, we obtain a connex, which has a $\nabla$-immersion from a primitive spherical connex since the images of $\tilde{z}_{0}, \alpha\left(\tilde{z}_{0}\right)$ and the two connected components of $\tilde{Z}-G\left\{\tilde{z}_{0}\right\}$ remain essential (see Figure 26-2).

Thus we obtain a $\nabla$-immersion from a spherical connex to $\mathscr{C}$. Now our lemma is verified. 
Lemma 7-2. Assume $\alpha(\tilde{Z}) \neq \tilde{Z}$ and that there is no place $\left(i, \tilde{z}_{i}\right)$ of type C satisfying $\tilde{Z}_{1-i}^{*} \subset \alpha(Z)$. Let $\tilde{L}$ be a connected subset of $\tilde{Z}$ such that $|\tilde{L}|$ is 1 or 2. Then there exists a place $\mathfrak{p}=\left(i, \tilde{z}_{i}\right)$ of type A satisfying $\tilde{L} \subset \tilde{F}_{\mathfrak{p}}^{*}$ and

(立) $\tilde{L} \cup\left\{\tilde{z}_{i}\right\} \not \subset \tilde{Z}_{1-i}$ if $\tilde{L} \cup\left\{\tilde{z}_{i}\right\}$ is connected.

Proof. We have to find only a place $\mathfrak{p}=\left(i, \tilde{z}_{i}\right)$ of type A or B satisfying $\tilde{L} \subset \tilde{F}_{p}^{*}$ and the property $(\tilde{L})$, because we find another place satisfying the desired properties by Lemma 6-9 if such a place $\mathfrak{p}$ of type B is given (see Figure 27).

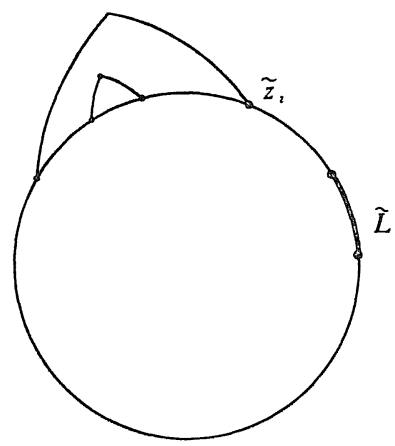

Figure 27.

First suppose $\alpha(\tilde{Z}) \cap \tilde{Z} \neq \varnothing$. Then we choose a point $\tilde{z}$ in a connected component of $\tilde{Z}-\alpha(\tilde{Z})$ disjoint to $\tilde{L}$ by Lemma $6-2$. We choose $i \in\{0,1\}$ so that $(i, \tilde{z})$ is a place satisfying $(\tilde{L})$ by Lemma $6-10$. If $(i, \tilde{z})$ is not of type $C$, then we put $\mathfrak{p}=(i, \tilde{z})$. If $(i, \tilde{z})$ is of type $\mathrm{C}$, then we put $\mathfrak{p}=\left(1-i, \tilde{z}_{1-i}\right)$. In any way, $\tilde{L} \subset \tilde{F}_{i}^{*}$ and the property $(\tilde{L})$ is satisfied (see Figure $28-1$ ).

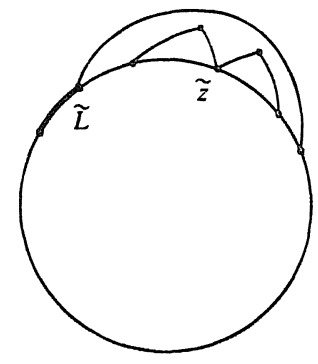

Figure 28-1.

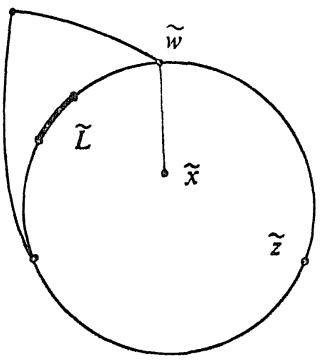

28-2.

Next suppose $\alpha(\tilde{Z}) \cap \tilde{Z}=\varnothing$. We take a point $\tilde{w}$ of $\tilde{Z}-\tilde{L}$ and choose $j \in$ $\{0,1\}$ so that $q=(j, \tilde{w})$ is a place satisfying $(\tilde{L})$ by Lemma 6-10, If $q$ is of type 
C, then we take another point of $\tilde{Z}-\tilde{L}$ adjacent to $\tilde{w}$ to obtain a place of type A or B by Lemma $6-8$, because $|\tilde{Z}| \geqq 4$ by Lemma 4-6. Then we may assume that $\mathfrak{q}$ is not of type C. If $\tilde{L} \subset \tilde{F}_{q}^{*}$, then we put $\mathfrak{p}=\mathfrak{q}$. We assume

$$
\tilde{L} \not \subset \tilde{F}_{q}^{*},
$$

where we may assume that $q$ is chosen so that the connected component $\tilde{S}$ of $\tilde{Z}-\tilde{F}_{q}$ intersecting $\tilde{L}$ is maximal. We take a non-terminal point $\tilde{z}$ of $\tilde{Z}-\tilde{S}$ and choose $k \in\{0,1\}$ so that $(k, \tilde{z})$ is a place satisfying $(\tilde{L})$ by Lemma $6-10$. If $(k, \tilde{z})$ is not of type $\mathrm{C}$, then we put $\mathfrak{p}=(k, \tilde{z})$ (see Figure 29).

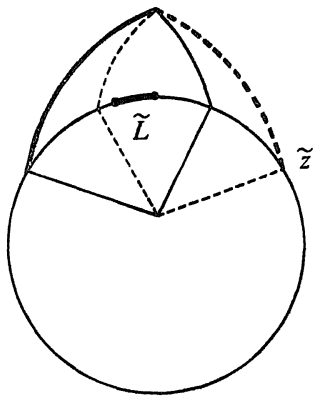

Figure 29-1.

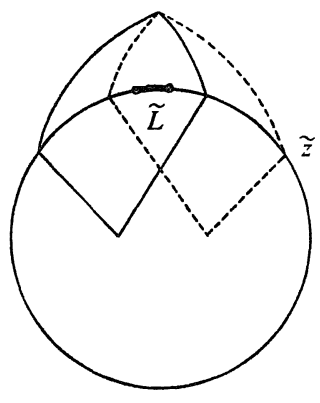

29-2.

If $(k, \tilde{z})$ is of type $\mathrm{C}$, then we put $\mathfrak{p}=\left(1-k, \tilde{z}_{1-k}\right)$ for $\tilde{z}_{1-k} \in \tilde{Z}_{1-k}^{*}$, where $\mathfrak{p}$ is a place of type A satisfying $(\tilde{L})$ and $\tilde{z}_{1-k}$ is a non-terminal point of $\tilde{Z}-\tilde{S}$ by Lemma 6-5 and Lemma 6-8. Thus we have

$$
\tilde{L} \subset \tilde{F}_{\mathfrak{p}}^{*}
$$

by Lemma 6-6 and the fact that $\tilde{S}$ is maximal (see Figure 28-2). Now our lemma is verified.

Lemma 7-3. Assume $\alpha(\tilde{Z}) \neq \tilde{Z}$ and that there is no place $\left(i, \tilde{z}_{i}\right)$ of type $\mathrm{C}$ satisfying $\tilde{Z}_{1-i}^{*} \subset \alpha(\tilde{Z})$. Let $\tilde{C}$ be a connected subset of $\tilde{X}-\tilde{F}$. Then $\operatorname{Supp}_{\tilde{F}}(\tilde{C})$ is contained in one of the following two types of sets:

$\nabla)$ complete set of at most three points,

$\square) g\left\{\tilde{u}_{0}, \tilde{u}_{1}, \tilde{x}_{0}, \tilde{x}_{1}\right\}$ where $g \in G, \tilde{u}_{j}$ is a point of $\tilde{Z}_{j}^{*}$ for $j=0,1$ and $\tilde{u}_{1}$ is adjacent to $\tilde{u}_{0}$.

Proof. We put $\tilde{S}=\operatorname{Supp}_{\tilde{F}}(\tilde{C})$. As $f(\tilde{S})$ is a complete subset of $\tilde{X}^{\prime \varepsilon}$ we may assume that there exists $g \in G, j \in\{0,1\}$ and points $\tilde{u}$ and $\tilde{v}$ of $\tilde{Z}_{j}$ such that $\tilde{v}$ is adjacent to $\tilde{v}$ and 


$$
\tilde{S} \subset g\left\{\tilde{u}, \tilde{v}, \tilde{x}_{0}, \tilde{x}_{1}\right\} .
$$

For conveniences sake we assume $g=1$. We assume also that $\tilde{S}$ contains $\tilde{x}_{1-j}$ and a point of $\tilde{Z}_{j}$. We put $\tilde{L}=\tilde{S} \cap \tilde{Z}$ and choose a place $p=\left(i, \tilde{z}_{i}\right)$ of type $A$ satisfying $\tilde{L} \subset \widetilde{F}_{p}^{*}$ and the property $(\tilde{L})$ by the above lemma. We apply Lemma 6-11 for $\mathfrak{p}$. Then iii) is impossible and ii) implies $\square$ ) by our hypothesis on $\mathfrak{p}$.

First suppose $i=j$. Then i) is impossible, because $\tilde{x}_{1-j} \in \widetilde{F}_{p}^{*}-\tilde{Z}$. By Lemma $6-10 \tilde{S}$ is a complete set of at most three essential points if $f_{\mathfrak{p}}(\tilde{S})$ is so.

Next suppose $i \neq j$. Then i) implies that $\tilde{S} \cap \tilde{Z}$ consists of a terminal point of $\widetilde{F}_{\mathfrak{p}}^{*}$. Hence $\widetilde{S}$ is a complete set of at most three points. Suppose that there exists a point, say $\tilde{u}$, of $\tilde{S} \cap \tilde{Z}_{1-i}^{*}$ and that $f_{\mathfrak{p}}(\tilde{S})$ is a complete subset of $\tilde{X}_{\mathfrak{p}}^{\varepsilon}$. Then $\tilde{u}$ is adjacent to $\tilde{z}_{i}$ and $\tilde{z}_{i} \notin \tilde{Z}_{1-i}$ by the property $(\tilde{L})$. If $\tilde{L}=\{\tilde{u}\}$, then $\left.\square\right)$ holds. Suppose $\tilde{L} \ni \tilde{v}$. Then $\tilde{v}$ is adjacent to $\tilde{x}_{i}$ since $|\tilde{Z}| \geqq 4$ by Lemma 4-6. Thus we obtain $f_{\mathfrak{p}}\left(\tilde{x}_{1-i}\right)$ is negligible by Lemma 4-1 and Lemma 6-10 (see Figure 30).

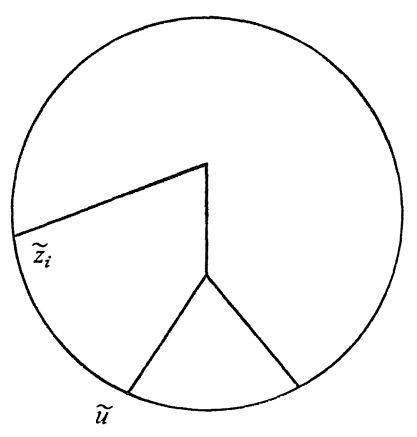

Figure 30.

This contradicts the fact that $\mathfrak{p}$ is of type A. Now our lemma is verified.

Lemma 7-4. Assume $\alpha(\tilde{Z}) \neq \tilde{Z}$ and that there is no place $\left(i, \tilde{z}_{i}\right)$ of type $\mathrm{C}$ satisfying $\tilde{Z}_{1-i}^{*} \subset \alpha(\tilde{Z})$. Then $\mathscr{C}$ has a $\nabla$-immersion from a spherical connex.

Proof. By the above lemma we have to determine only $\bar{W}_{\tilde{F}}\left(\left\{\tilde{u}_{0}, \tilde{u}_{1}, \tilde{x}_{0}, \tilde{x}_{1}\right\}\right)$ for $\left(\tilde{u}_{0}, \tilde{u}_{1}\right) \in \tilde{Z}_{0}^{*} \times \tilde{Z}_{1}^{*}$ such that $\tilde{u}_{1}$ is adjacent to $\tilde{u}_{0}$. We put $\tilde{L}=\left\{\tilde{u}_{0}, \tilde{u}_{1}\right\}$ and choose a place $\mathfrak{p}=\left(i, \tilde{z}_{i}\right)$ of type A satisfying $\tilde{L} \subset \tilde{F}_{p}^{*}$ and the property $(\tilde{L})$ by Lemma 7-2. Then we obtain a $\nabla$-immersion from a primitive spherical connex to $\mathscr{C}_{p}$, where

$$
\left\{\tilde{u}_{0}, \tilde{u}_{1}, \tilde{x}_{0}, \tilde{x}_{1}\right\} \subset \tilde{F}_{p}
$$

and $f_{\mathfrak{p}}$ is injective on $\bar{W}_{\bar{F}}\left(\left\{\tilde{u}_{0}, \tilde{u}_{1}, \tilde{x}_{0}, \tilde{x}_{1}\right\}\right)$. 
We obtain a $\nabla$-immersion from a spherical connex to $\mathscr{C}$, triangulating the rectangles by the above method (see Figure 31).

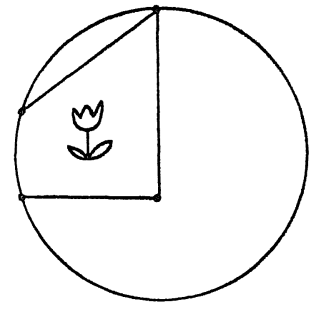

Figure 31-1.

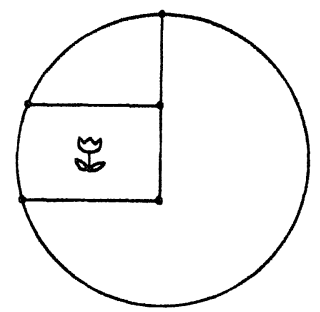

31-2.

Lemma 7-5. Assume $\alpha(\tilde{Z}) \neq \tilde{Z}$ and that there exists a place $\mathfrak{p}=\left(i, \tilde{z}_{i}\right)$ of type $\mathrm{C}$ such that $\tilde{Z}_{1-i}^{*} \subset \alpha(\tilde{Z})$. Let $\tilde{C}$ be a connected subset of $\operatorname{Web}_{\tilde{F}}\left(\tilde{Z} \cup\left\{\tilde{x}_{0}\right.\right.$, $\left.\left.\tilde{x}_{1}\right\}\right)$. Then $\operatorname{Supp}(\tilde{C})$ is complete or

$$
\operatorname{Supp}_{\tilde{F}}(\tilde{C}) \subset\left\{\tilde{x}_{0}, \tilde{x}_{1}, \tilde{z}_{i}, \tilde{z}_{1-i}, \tilde{z}\right\},
$$

where $\tilde{z}_{1-i} \in \tilde{Z}_{1-i}^{*}$ and $\tilde{z}$ is the terminal point of $\tilde{Z}_{i}$ besides $\tilde{z}_{i}$.

Proof. If $\tilde{x}_{1-i} \notin \operatorname{Supp}_{\tilde{F}}(\tilde{C})$, then our assertion is obvious. Suppose $\tilde{x}_{1-i} \in \operatorname{Supp}_{\tilde{F}}(\tilde{C})$. Then $\operatorname{Supp}_{\tilde{F}}(\tilde{C})$ has no non-terminal point of $\tilde{Z}_{i}$ by Lemma 6-11 (see Figure 32).

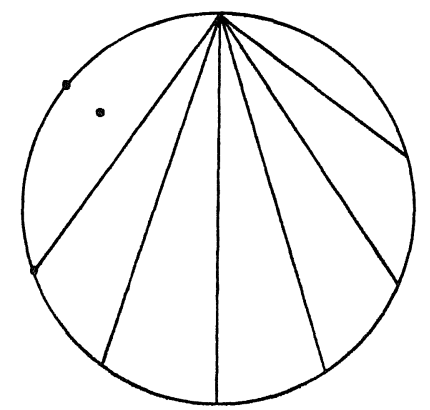

Figure 32.

Lemma 7-6. Assume $\alpha(\tilde{Z}) \neq \tilde{Z}$ and that there exists a place $\mathfrak{p}=\left(i, \tilde{z}_{i}\right)$ of type $\mathrm{C}$ such that $\tilde{Z}_{1-i}^{*} \subset \alpha(\tilde{Z})$. Then $\mathscr{C}$ has a $\nabla$-immersion from a spherical connex.

Proof. By the above lemma we have to determine only $\bar{W}_{\tilde{F}}\left(\left\{\tilde{x}_{0}, \tilde{x}_{1}, \tilde{z}_{i}\right.\right.$, $\left.\left.\tilde{z}_{1-i}, \tilde{z}\right\}\right)$ where $\tilde{z}_{1-i}$ is the sole point of $\tilde{Z}_{1-i}^{*}$ and $\tilde{z}$ is the terminal point of $\tilde{Z}_{i}$ 
besides $\tilde{z}_{i}$. Let $\tilde{S}$ and $\tilde{T}$ be the connected components of $\tilde{Z}-\left\{\tilde{z}_{1-i}, \alpha\left(\tilde{z}_{1-i}\right)\right\}$. Then we define a contraction $f_{\mathrm{c}}$ so that the inverse image of a point under $f_{\mathrm{c}}$ is a point or one of the followings:

$$
\begin{aligned}
& g\left\{\tilde{x}_{i}, \alpha\left(\tilde{z}_{1-i}\right)\right\} \\
& g\left(\bar{W}_{F}\left(\tilde{S} \cup \alpha(\tilde{T}) \cup\left\{\tilde{z}_{1-i}, \alpha\left(\tilde{z}_{1-}\right)\right\}\right)-\left\{\tilde{z}_{1-i}, \alpha\left(\tilde{z}_{1-i}\right)\right\}\right)
\end{aligned}
$$

where $g \in G$ (see Figure 33).

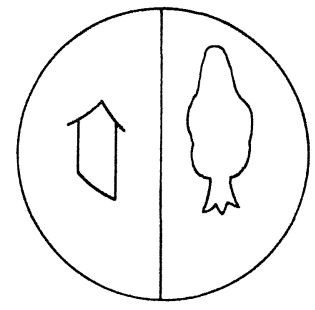

Figure 33-1.

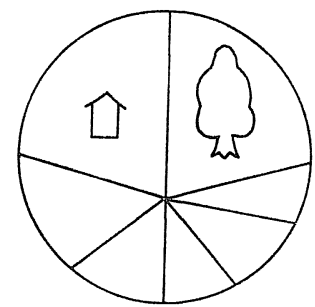

$33-2$.

We obtain a $\nabla$-immersion from a spherical connex to $\mathscr{C}$, triangulating the rectangle by the above method. Now our lemma is verified.

Proof of Theorem 4. Let the assumptions be as in the beginning of this section. Then $\mathscr{C}$ has a $\nabla$-immersion $\iota_{1}$ from a spherical connex $\mathscr{C}_{1}$ by Lemma 7-1, Lemma 7-4 and Lemma 7-6. On the other hand $\mathscr{C}_{1}$ has an immersion $c_{0}$ from a primitive connex $\mathscr{C}_{0}$ by Theorem 2 , which is tame.

Suppose first that $\mathscr{C}$ is primitive. Then $\mathscr{C}$ is isomorphic to $\mathscr{C}_{1}$.

Next suppose that $\mathscr{C}$ is not primitive. Then $\mathscr{C}_{0}$ has a $\nabla$-immersion from a primitive spherical connex by induction hypothesis, therefore $\mathscr{C}_{0}$ is spherical. As $\mathscr{C}_{0}$ has only one embedding into $S^{2}$ in topological sense the triangulation according to $\mathscr{C}_{0}$ is a refinement of the one according to $\mathscr{C}_{1}$. Therefore $\iota_{0}$ is a $\nabla$-immersion (see Figure 34).

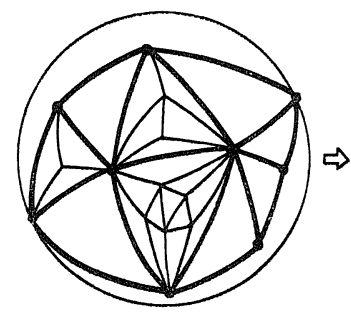

Figure 34-1.

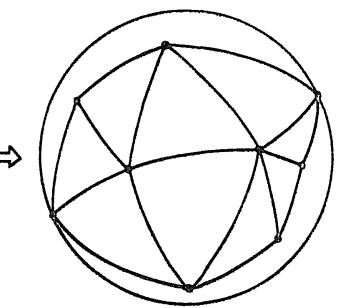

$34-2$. 
Now $\mathscr{C}$ has a $\nabla$-immersion $\iota_{1}{ }^{\circ} \iota_{0}$ from the primitive spherical connex $\mathscr{C}_{0}$.

\section{References}

[1] Lehman, A., A solution of the Shannon switching game, J. Soc. Ind. Appl. Math., 12 (1964), 687-725.

[2] Yamasaki, Y., Theory of division games, Publ. RIMS, Kyoto Univ., 14 (1978), $337-$ 358.

[ 3 ] —, Theory of connexes. II, in preparation. 